\title{
Synthetic utility of glycosyl triazoles in carbohydrate chemistry
}

Brendan L. Wilkinson, ${ }^{a}$ Laurent F. Bornaghi, ${ }^{a}$ Sally-Ann Poulsen ${ }^{a}$ and Todd A. Houston ${ }^{b, *}$

${ }^{a}$ Eskitis Institute for Cell and Molecular Therapies, Griffith University, 170 Kessels Road, Nathan, Queensland 4111, Australia

${ }^{b}$ Institute for Glycomics, Griffith University, PMB 50 Gold Coast Mail Centre, Gold Coast, Queensland 9726, Australia

"Corresponding author: Tel.: +61 73735 4115; Fax: +71 73735 7656; e-mail:

T.Houston@griffith.edu.au 


\section{Table of Contents Graphic}

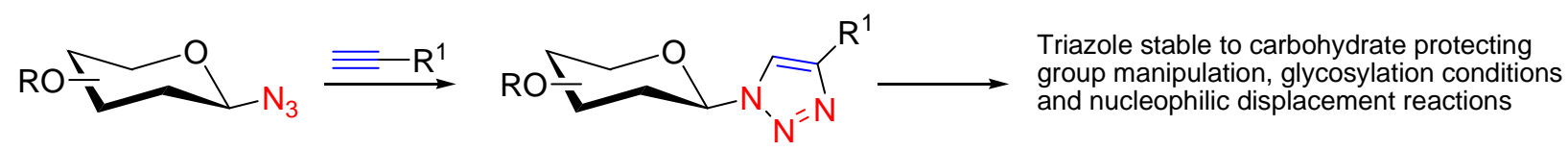




\begin{abstract}
We report herein a study of the synthetic utility of the glucosyl triazole moiety in carbohydrate chemistry. A model glucosyl triazole was prepared by a modified Huisgen 1,3-dipolar cycloaddition reaction. The relative rate of cycloaddition was investigated using a variety of alcohol co-solvents and reaction temperatures. It was found that the reaction proceeded with similar efficiency irrespective of co-solvent, however mildly elevated temperatures $\left(40{ }^{\circ} \mathrm{C} \mathrm{cf} . \mathrm{rt}\right)$ increased the speed of reaction significantly $(2 \mathrm{~h} \mathrm{cf.} 8 \mathrm{~h})$. The robustness of the triazole moiety was then interrogated under conditions typically encountered in carbohydrate chemistry reaction sequences - alcohol group protection/deprotection, nucleophilic displacement and $O$ glycosylation. The triazole integrity was retained in all cases studied as evidenced from full compound characterization. Finally, a diverse set of triazole-linked glycoconjugates was synthesized. Collectively our results demonstrated that the glucosyl triazole moiety was indeed a robust entity for carbohydrate chemistry.
\end{abstract}

\title{
Keywords
}

Carbohydrate; Glycoconjugate; Huisgen reaction; Click chemistry

\section{Introduction}

Oligosaccharides linked to proteins and lipids via $\mathrm{N}$ - or $\mathrm{O}$-glycosidic linkages (glycoconjugates) have been shown to govern crucial life processes and disease states. ${ }^{1-7}$ Glycosylation patterns for 
proteins and lipids are exquisitely controlled via mechanisms independent from that of the genomic transcription and as a result glycoconjugates from natural sources often exist as heterogenous isoforms (glycoforms). The isolation of homogenous material from crude biological extracts in sufficient quantities for study can, therefore, be difficult. Medicinal chemistry, through provision of an impressive array of synthetic methodologies, offers a potentially more reliable route to homogenous glycoconjugates leading to either exact copies of naturally occurring glycoconjugates or, alternatively, incorporating unnatural glycosidic linkages. An appreciable benefit of the latter approach is that the 'artificial' glyconjugate may retain the geometric and spatial characteristics of the native glycoform ${ }^{8,9}$ yet exhibit stability towards $N$ and $O$-glycosyl hydrolase activity and are able to inhibit these enzymes to some extent, ${ }^{10}$ so increasing the potential for a wide array of in vivo applications. In addition the artificial linkage may be fine tuned as an inert functionality towards subsequent synthetic transformations necessary elsewhere within the target molecule synthesis.

In the past three years there has been a flourish of activity in the literature concerning the 1,3dipolar cycloaddition reaction (1,3-DCR) of organic azides with terminal acetylenes (Huisgen reaction). ${ }^{11}$ This interest stems largely from the optimisation of $1,3-\mathrm{DCR}$ by Sharpless ${ }^{12}$ and Melda ${ }^{13}$ with respect to ease and efficiency of catalysis and regioselectivity of the triazole product. The reaction involves a step-wise $\mathrm{Cu}(\mathrm{I})$-catalyzed dipolar cycloaddition of a terminal acetylene to an organic azide to form, exclusively, the 1,4-disubstituted-1,2,3-triazole (Scheme 1). ${ }^{12,13}$ The highly exothermic and kinetically controlled reaction is conducted favorably in water and is also tolerant to a wide variety of organic co-solvents and as such has become a premier component of "click chemistry". $12 \mathrm{~b}$ 


\section{Insert Scheme 1}

The modified 1,3-DCR has shown great versatility and utility on non-saccharide substrates in medicinal chemistry, chemical biology and materials science. ${ }^{14,15}$ The application to "traditional" carbohydrate chemistry ${ }^{16}$ has been somewhat slower, but presents as an attractive reaction for several reasons. Glycosyl azides are generally stable, crystalline solids, inert towards a wide range of reaction conditions and are available diastereomerically pure. $^{17}$ In traditional carbohydrate synthesis they serve primarily as stable precursors of glycosylamines (for glycopeptide synthesis) and are also less commonly precursors to glycosylfluoride donors. ${ }^{18}$ Glycosyl azides are readily synthesized through the stereoselective bimolecular displacement of a glycosyl halide with a an azide nucleophile ${ }^{17}$ and 2-azido sugars are obtained through the triflyl azide-mediated diazotransfer of amino sugars. ${ }^{19}$ Preparation of carbohydrates containing a terminal acetylene moiety is also readily achieved either by the alkylation of carbohydrates (to synthesize propargyl ethers) or Lewis-acid-catalyzed glycosylation (to synthesize O-propargyl glycosides). ${ }^{20}$ The 1,3-DCR utilizes low-cost and relative non-toxic reagents and solvents so alleviating the need to use often expensive and highly toxic glycosylation and peptide coupling reagents, the risk and costs of which are acute on bulk scales. Finally, product purification is simple, in many cases chromatography is not necessary with either precipitation ${ }^{21}$ or liquid-liquid extraction sufficient to obtain pure product.

The incorporation of an azide and/or an alkyne moiety on a carbohydrate scaffold unleashes the potential to access a new dimension of structural diversity to complement the vast structural 
diversity already inherent to carbohydrates and so it is anticipated that interest in the 1,3-DCR with carbohydrate substrates will grow. A detailed synthetic analysis concerning the utility of this reaction and the stability of the formed glycosyl triazole linkage towards typical carbohydrate reaction sequences would be beneficial to assess the viability of the glycosyl triazole linker as a tool for carbohydrate chemistry, and was the inspiration for the study described herein. Specifically, we report (i) an investigation of the rate of formation of the glycosyl triazole linkage with variable solvent and temperature parameters, (ii) the stability of this linkage towards conditions commonly used in carbohydrate reaction sequences, including hydroxyl group protection/deprotection, $O$-glycosylations and nucleophilic displacement, (iii) an examination of the retention of anomeric stereochemistry and the rates of triazole formation with respect to the glycosyl azide precursor configuration $(\alpha / \beta)$, and (iv) the versatility of the 1,3-DCR of a various sugar azides with a diverse array of acetylene partners.

\section{Results and discussion}

The model glycosyl triazole employed to investigate the study of rate of triazole formation was the glucosyl triazole compound $2 .{ }^{16 \mathrm{f}}$ Compound 2 was prepared by $1,3-\mathrm{DCR}$ of propargyl alcohol with 2,3,4,6-tetra-O-acetyl- $\beta$-D-glucopyranosyl azide (1) using a procedure adapted from literature. $^{12,13}$ Reaction of propargyl alcohol with the azide partner $\mathbf{1}$ in the presence of a $\mathrm{CuSO}_{4} /$ ascorbate mixture in aqueous alcohol, affords exclusively the 1,4-disubstituted $\beta$-Dglucosyltriazole $\mathbf{2}$ with complete retention of anomeric stereochemistry and in high yield. This configuration has been confirmed by X-ray crystallography. ${ }^{22}$ The effect of alcohol co-solvents methanol, ethanol, isopropanol, tert-butanol and propargyl alcohol on reaction completion time is 
presented in Table 1. Irrespective of the co-solvent employed, the reaction to form $\mathbf{2}$ was complete within $2 \mathrm{~h}$ (as evidenced by TLC) when the reaction was carried out at a slightly elevated temperature $\left(40{ }^{\circ} \mathrm{C}\right)$ (Table 1 , entries $\left.1-5\right)$. Noteworthy is that when the coupling partner propargyl alcohol was employed also as bulk co-solvent this did not substantially increase the rate of reaction. An increased reaction time $(8 \mathrm{~h})$ was necessary to afford complete conversion when the reaction was conducted at room temperature (Table 1, compare entries 4 and 6), although the yields were similar (90 cf. 92\%). The reactants were initially insoluble in all solvent media, but as the reaction progressed, a deep yellow, homogenous mixture was observed. In all cases the yield (following liquid-liquid extraction into $\mathrm{CH}_{2} \mathrm{Cl}_{2}$ ) was high and exhibited minimal variability (79-92\%). This simple work up provided material sufficiently pure for most ensuing synthetic purposes, however a final solid-phase extraction (SPE) purification step was employed to remove trace paramagnetic $\mathrm{Cu}$ (II) salts for the purpose of NMR analysis and the provision of analytically pure material. Reaction conditions were simple, requiring only vigorous stirring at the designated temperature within capped scintillation vials under air.

\section{Insert Table 1}

Next, the stability of the glycosyl triazole linkage towards commonly used carbohydrate chemistry reaction conditions was investigated (Scheme 2). Silyl, trityl, acetyl, benzoyl and benzyl alcohol protecting groups were all incorporated smoothly on to the alcohol moiety of $\mathbf{2}$ to generate compounds 3-7, respectively, with the triazole linkage remaining intact. Reaction conditions to remove these protecting groups were also compatible with the triazole linkage, either regenerating 2 or the globally deprotected analogue 11. Protection and deprotection yields 
were excellent with no baseline decomposition observed by TLC. Interestingly, the hydrogenation of the benzylated compound 7 was sluggish using typical conditions $\left(\mathrm{H}_{2}, 5 \%\right.$ $\mathrm{Pd} / \mathrm{C}$ ), necessitating basic hydrogenation conditions (ie. $\mathrm{H}_{2}, 30 \% \mathrm{Pd}\left(\mathrm{OH}_{2}\right) / \mathrm{C}$ ). Removal of the benzyl ether in the 2 position of the sugar was potentially problematic, quite possibly due to steric hindrance imposed by the glucosyl triazole. Despite this, the free glucosyl triazole 11 was recovered in good yield (78\%) after $24 \mathrm{~h}$. Glycosylation of the triazole acceptor 2 using either typical Lewis acid-catalysis $\left(\mathrm{BF}_{3} \cdot \mathrm{Et}_{2} \mathrm{O}\right)$ or Knoenigs-Knorr conditions (AgOTf) successfully generated 8 and $\mathbf{9}$, respectively, although yields were not high (53\% and $41 \%$, respectively). This was possibly due to the weak glycosyl acceptor nature of the triazole alcohol. Alternatively, the triazole may interfere with activation of the glycosyl donor through interaction with the Lewis acid; nonetheless, this provides rapid entry into disaccharide mimics. Finally, the azide displacement of the crude mesylated intermediate from $\mathbf{2}$ to form the triazole azide $\mathbf{1 0}$ provides a system for carrying out iterative click reactions.

\section{Insert Scheme 2}

Having established some of the chemical modifications available, we set out to synthesize a variety of glycoconjugates with diversity in both the carbohydrate and aglycone moieties. Derivatives of hydrophobic bioactive compounds and synthetic precursors to more complex glycoconjugates were sought as part of our general focus on carbohydrate-based therapeutics. Table 2 shows compounds synthesized from our model glucosyl azide 1 (compounds 12-15), methyl 2,3,4-tri-O-acetyl-1-azido-1-deoxy- $\beta$-D-glucopyranuronate ${ }^{23}$ (compound 16), 2,3,4-tri-Oacetyl- $\alpha$-D-arabinopyranosyl azide (compound 17), 2-acetamido-3,4,6-tri-O-acetyl-2-deoxy- $\beta$-Dglucopyranosyl azide (compound $\mathbf{1 8}^{16 f}$ ) and 2,3,4,6-tetra- $O$-benzyl- $\alpha$-D-glucosyl azide 
(compound 19). Minimal or no chromatography was required in all cases to obtain samples of sufficient purity for further synthetic transformation. Reaction rates and yields varied somewhat depending on the acetylene dipolarophilicity and solubility. While the reaction is reported as reasonably insensitive to substrate solubility, more hydrophobic substrates generally took longer to react, as is shown for the steroidal glucosyl triazole 15. Interestingly, while the reaction for $\mathbf{1 5}$ took $20 \mathrm{~h}$ to reach completion, the estradiol analogue 14 required only 30 minutes for completion under identical reaction conditions. Furthermore, both compounds were isolated by precipitation from cold water, although a final chromatographic purification step was required for these two compounds in order to remove traces of starting materials and $\mathrm{Cu}$ salts.

Our attention then shifted to examining the rate dependence on anomeric configuration and the nature of the saccharide protecting groups. The reaction of 2,3,4,6-tetra-O-benzyl- $\alpha$-D-glucosyl azide to the $\alpha$-glucosyl triazole 19 proceeded with complete $\alpha$-retention of anomeric stereochemistry. The reaction was slow, requiring a total of $c a .48 \mathrm{~h}$ at $60{ }^{\circ} \mathrm{C}$ to reach completion. Doubling of the $\mathrm{Cu}(\mathrm{II})$ and ascorbate catalyst load (ie 0.4 equiv $\mathrm{Cu}(\mathrm{II}), 0.8$ equiv ascorbate) was also required since gradual oxidation of the $\mathrm{Cu}(\mathrm{I})$-complex intermediate was observed using standard conditions and no conversion was observed after $8 \mathrm{~h}$ by either TLC or ESI-MS. The sluggish nature of this reaction was rationalized as resulting from increased steric bulk surrounding the anomeric center upon transformation from the linear azide to the triazole, increased hydrophobicity imparted by the benzyl groups, and stabilization of the azide dipole by the anomeric effect.

\section{Insert Table 2}


The scope of the reaction can be expanded further by installing the azide/acetylene functionality into other positions on the carbohydrate ring and/or on to different carbohydrate partners to serve as a convenient and direct route to triazole-tethered disaccharide mimics. The triazole-tethered disaccharide $\mathbf{2 0}$ was formed in 78\% yield from the 1,3-DCR of a 6-azido glucose analogue and a glucose-derived propargyl ether. This yield is an improvement over the glycosylations on $\mathbf{2}$ to synthesize the structurally related triazole-tethered disaccharides $8(52 \%)$ and $9(43 \%) .1,3-\mathrm{DCR}$ can therefore serve as a useful means of rapidly generating artificial cyclodextrins, ${ }^{24}$ glycodendrimers ${ }^{25}$ and glycopolymers ${ }^{26}$ under kinetically controlled conditions.

\section{Insert chemical structure of compound 20}

\section{Conclusions}

The facile $\mathrm{Cu}(\mathrm{I})$-catalyzed 1,3-dipolar cycloaddition reaction has emerged of late as a potent tool in accessing diverse molecular architectures. Its versatility and potential is now starting to be realized in glycochemistry, providing expedient access to an interesting and relatively new family of pharmacologically relevant heterocyclic carbohydrates. Owing to the inherent complex nature of carbohydrate chemistry, especially concerning anomeric stereochemistry and stability, the reaction has not been sufficiently scrutinized as a viable alternative or addition to classical methods. We have successfully explored the utility of the reaction by examining the compatibility and tolerance of the conditions to pre-installed saccharide protecting groups, but also the stability of the installed triazole linkage under protection/deprotection sequences and glycosylations. The reaction is forgiving, mild, high yielding, simple to purify, and stereo- and 
regiospecific. Owing to such impressive versatility, we envisage the reaction to be useful addition to the arsenal of traditional solution-based reactions within carbohydrate chemistry. Further exploration of the scope of this chemistry will also include modification of other hydrophobic drug molecules to improve their water solubility. ${ }^{27}$ Furthermore, due to the inherent robustness of the glycosyl triazole linkage, it is reasonable to expect the reaction to be compatible with existing solution- and solid-phase, convergent (block) and linear glycoprotein/peptide strategies. Finally, recent regioselective access to the 1,5-disubstituted triazole from azide and alkyne precursors will expand the structural diversity available from the chemistry presented here. ${ }^{28}$

\section{Experimental}

\section{General}

Glycosyl azide precursors were prepared by phase transfer nucleophilic displacement of corresponding peracetylated $\alpha$-glycosylbromide. ${ }^{17}$ All reagents were purchased from commercial sources and were used without further purification. All solvents were available commercially dried or freshly dried and distilled prior to use. ${ }^{1} \mathrm{H}$ NMR (400 MHz), ${ }^{13} \mathrm{C}$ NMR (100 MHz), 2D gCOSY and gHSQC spectra were recorded on a $400 \mathrm{MHz}$ spectrometer with chemical shift values given in ppm $(\delta)$ relative to TMS $(0.00 \mathrm{ppm})$ using $\mathrm{CDCl}_{3}$ solvent unless otherwise stated. Melting points are reported as uncorrected. High resolution electrospray ionisation mass spectra (HRESIMS) were recorded on a 4.7T Fourier transform mass spectrometer in positive ion mode unless otherwise stated. Reaction progress was monitored by TLC using Silica gel-60 F 254 plates with detection by short wave UV fluorescence $(\lambda=254 \mathrm{~nm})$ and staining with $10 \%(\mathrm{v} / \mathrm{v}) \mathrm{H}_{2} \mathrm{SO}_{4}$ in ethanol char. Flash chromatography was conducted using flash silica gel (60 - 240 mesh). Solid phase extraction (SPE) was conducted using cartridges prepacked with silica sorbent. 


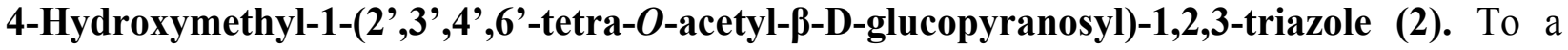
vigorously stirring suspension of 2,3,4,6-tetra-O-acetyl- $\beta$-D-glucopyranosyl azide $(2.0 \mathrm{~g}, 5.35$ mmol) in tert-butyl alcohol $(11 \mathrm{~mL})$ was added propargyl alcohol $(1.3 \mathrm{~mL}, 22.43 \mathrm{mmol}, 4.2$ equiv). The reaction was initiated by the addition of a solution of $\mathrm{CuSO}_{4} \cdot 5 \mathrm{H}_{2} \mathrm{O}(270 \mathrm{mg}, 1.08$ mmol, 0.2 equiv) and sodium ascorbate (424.5 mg, $2.14 \mathrm{mmol}, 0.4$ equiv) in distilled $\mathrm{H}_{2} \mathrm{O}$ (11 $\mathrm{mL}$ ). The deep yellow suspension was stirred vigorously at $40{ }^{\circ} \mathrm{C}$ for $2 \mathrm{~h}$. At this time, TLC indicated reaction completion (1:1 ethyl acetate:hexanes). Distilled $\mathrm{H}_{2} \mathrm{O}(20 \mathrm{~mL})$ was added and the aqueous layer extracted twice with $\mathrm{CH}_{2} \mathrm{Cl}_{2}(2 \times 50 \mathrm{~mL})$. The combined organic extracts were dried $\left(\mathrm{MgSO}_{4}\right)$, filtered and evaporated to afford a crude yellow solid residue which was recrystallized from hot absolute ethanol to afford the triazole $\mathbf{1}$ as off-white crystalline solid (2.1 g, 92\%). Mp: $150-151{ }^{\circ} \mathrm{C}$ (Lit. Mp: $148-150{ }^{\circ} \mathrm{C}$ as a mixture of 4- and 5-hydroxymethyl regioisomers $\left.^{16 f}\right) . R_{f}=0.22$ (8:2 ethyl acetate:hexanes). ${ }^{1} \mathrm{H}$ NMR $\left(400 \mathrm{MHz}, \mathrm{CDCl}_{3}\right): \delta 1.87(\mathrm{~s}$, 3H, OAc) $2.01(\mathrm{~s}, 3 \mathrm{H}, \mathrm{OAc}) 2.05(\mathrm{~s}, 3 \mathrm{H}, \mathrm{OAc}) 2.06(\mathrm{~s}, 3 \mathrm{H}, \mathrm{OAc}) 3.99\left(\mathrm{ddd},{ }^{3} \mathrm{~J}_{5-4}=10.2 \mathrm{~Hz},{ }^{3} \mathrm{~J}_{5-6}\right.$ ' $\left.=5.2 \mathrm{~Hz},{ }^{3} \mathrm{~J}_{5-6}=2.4 \mathrm{~Hz}, 1 \mathrm{H}, \mathrm{H}-5\right) 4.13\left(\mathrm{dd},{ }^{2} \mathrm{~J}_{6-6},=12.4 \mathrm{~Hz},{ }^{3} \mathrm{~J}_{6-5}=2.4 \mathrm{~Hz}, 1 \mathrm{H}, \mathrm{H}-6\right) 4.28\left(\mathrm{dd},{ }^{2} \mathrm{~J}_{6}\right.$ '${ }_{6}=12.4 \mathrm{~Hz},{ }^{3} \mathrm{~J}_{6}{ }^{\prime}-5=5.2 \mathrm{~Hz}, 1 \mathrm{H}, \mathrm{H}-6$ ') $4.79\left(\mathrm{~s}, 2 \mathrm{H}, \mathrm{CH}_{2} \mathrm{OH}\right) 5.20-5.25(\mathrm{~m}, 1 \mathrm{H}, \mathrm{H}-4) 5.38-5.45$ $(\mathrm{m}, 2 \mathrm{H}, \mathrm{H}-3 / \mathrm{H}-2) 5.86-5.88(\mathrm{~m}, 1 \mathrm{H}, \mathrm{H}-1) 7.78(\mathrm{~s}, 1 \mathrm{H}$, triazole $\mathrm{H}) ;{ }^{13} \mathrm{C}$ NMR $(100 \mathrm{MHz}$, $\mathrm{CDCl}_{3}$ ): $\delta 20.4(\mathrm{OAc}) 20.7$ (OAc) 20.75 (OAc) 20.9 (OAc) $56.6\left(\mathrm{CH}_{2} \mathrm{OH}\right) 61.8$ (C-6) 67.9 (C-2) 70.6 (C-3) 72.9 (C-4) 75.3 (C-5) 86.1 (C-1) 120.9 (triazole CH) 148.5 (triazole C) 169.3 (OAc) 169.6 (OAc) 170.2 (OAc) 170.7 (OAc). HRESIMS calculated for $\mathrm{C}_{17} \mathrm{H}_{23} \mathrm{~N}_{3} \mathrm{O}_{10} \mathrm{Na}^{+}: 452.127565$. Found: 452.126953. Anal. Calculated for $\mathrm{C}_{17} \mathrm{H}_{23} \mathrm{~N}_{3} \mathrm{O}_{10}$ : C, 47.55; H, 5.40; N, 9.79. Found: C, 47.33; H, 5.39; N, 9.51. 


\section{4-tert-Butyldimethylsilyloxymethyl-1-(2',3',4',6'-tetra-O-acetyl- $\beta$-D-glucopyranosyl)-1,2,3-}

triazole (3). A solution of the alcohol $2(200 \mathrm{mg}, 0.47 \mathrm{mmol})$ and imidazole $(95 \mathrm{mg}, 1.40 \mathrm{mmol}$, 3 equiv) was prepared in dry $\mathrm{CH}_{2} \mathrm{Cl}_{2}(5 \mathrm{~mL})$ under nitrogen and tert-butyldimethylsilyl chloride ( $85 \mathrm{mg}, 0.56 \mathrm{mmol}, 1.2$ equiv) was added in a single portion. The solution was stirred at rt under nitrogen for $2 \mathrm{~h}$ at which time, TLC indicated reaction completion (8:2 ethyl acetate/hexanes). $\mathrm{CH}_{2} \mathrm{Cl}_{2}(5 \mathrm{~mL})$ was then added and the solution was washed with $1 N \mathrm{HCl}(5 \mathrm{~mL})$, saturated $\mathrm{NaHCO}_{3}(5 \mathrm{~mL})$ and brine $(5 \mathrm{~mL})$. The organic layer was dried $\left(\mathrm{MgSO}_{4}\right)$, filtered and evaporated to a crude oil, which was purified by flash silica chromatography (4:6 ethyl acetate:hexanes) to afford a white solid (225 mg, $0.41 \mathrm{mmol}, 88 \%)$. Mp: $100-101{ }^{\circ} \mathrm{C} . R_{f}=0.74$ (8:2 ethyl acetate:hexanes). ${ }^{1} \mathrm{H}$ NMR (400 MHz, $\left.\mathrm{CDCl}_{3}\right): \delta 0.08\left(\mathrm{~s}, 6 \mathrm{H}, 2\right.$ x $\left.\mathrm{SiCH}_{3}\right) 0.90(\mathrm{~s}, 9 \mathrm{H}, t-\mathrm{Bu}) 1.85$ (s, 3H, OAc) $2.01(\mathrm{~s}, 3 \mathrm{H}, \mathrm{OAc}) 2.05(\mathrm{~s}, 3 \mathrm{H}, \mathrm{OAc}) 2.06(\mathrm{~s}, 3 \mathrm{H}, \mathrm{OAc}) 3.98\left(\mathrm{ddd},{ }^{3} \mathrm{~J}_{5-4}=10.4 \mathrm{~Hz}\right.$, $\left.{ }^{3} \mathrm{~J}_{5-6},=5.2 \mathrm{~Hz},{ }^{3} \mathrm{~J}_{5-6}=2.4 \mathrm{~Hz}, 1 \mathrm{H}, \mathrm{H}-5\right) 4.14\left(\mathrm{dd},{ }^{2} \mathrm{~J}_{6-6},=12.8 \mathrm{~Hz},{ }^{3} \mathrm{~J}_{6-5}=2.4 \mathrm{~Hz}, 1 \mathrm{H}, \mathrm{H}-6\right) 4.23$ $\left(\mathrm{dd},{ }^{2} \mathrm{~J}_{6^{\prime}-6}=12.8 \mathrm{~Hz},{ }^{3} \mathrm{~J}_{6}{ }^{\prime}-5=5.2 \mathrm{~Hz}, 1 \mathrm{H}, \mathrm{H} 6^{\prime}\right) 4.82\left(\mathrm{~s}, 2 \mathrm{H}, \mathrm{CH}_{2} \mathrm{OSi}\right) 5.21-5.26(\mathrm{~m}, 1 \mathrm{H}, \mathrm{H}-4) 5.37$ - $5.41(\mathrm{~m}, 1 \mathrm{H}, \mathrm{H}-3) 5.44-5.48(\mathrm{~m}, 1 \mathrm{H}, \mathrm{H}-2) 5.85\left(\mathrm{~d},{ }^{3} \mathrm{~J}_{1-2}=9.2 \mathrm{~Hz}, 1 \mathrm{H}, \mathrm{H}-1\right) 7.67(\mathrm{~s}, 1 \mathrm{H}$, triazole $\mathrm{CH}),{ }^{13} \mathrm{C}$ NMR $\left(100 \mathrm{MHz}, \mathrm{CDCl}_{3}\right): \delta-5.2\left(\mathrm{SiCH}_{3}\right)-5.1\left(\mathrm{SiCH}_{3}\right) 18.5(\mathrm{TBDMS}$ quart $\mathrm{C})$ 20.4 (OAc) 20.71 (OAc) 20.74 (OAc) 20.9 (OAc) 26.1 ( $t$-Bu) 57.9 ( $\left.\mathrm{CH}_{2} \mathrm{OTBDMS}\right) 61.8$ (C-6) 67.9 (C-4) 70.4 (C-3) 73.0 (C-2) 75.3 (C-5) 85.8 (C-1) 120.1 (triazole CH) 149.6 (triazole C) $169.1 \quad$ (OAc) $\quad 169.6 \quad$ (OAc) $170.1 \quad$ (OAc) $170.7 \quad$ (OAc). HRESIMS calculated for $\mathrm{C}_{23} \mathrm{H}_{37} \mathrm{~N}_{3} \mathrm{O}_{10} \mathrm{SiNa}^{+}$: 566.214042. Found: 566.215711. Anal. Calculated for $\mathrm{C}_{23} \mathrm{H}_{37} \mathrm{~N}_{3} \mathrm{O}_{10} \mathrm{Si}$ : C, 50.81; H, 6.86; N, 7.73; Found: C, 50.89; H, 7.07; N, 7.61.

Deprotection of silyl ether $\mathbf{3} \rightarrow \mathbf{2}$. A portion of the silyl ether $\mathbf{3}(25 \mathrm{mg}, 0.05 \mathrm{mmol})$ was then dissolved in dry THF $(1.0 \mathrm{~mL})$ and a $1.0 \mathrm{M}$ solution of tetrabutylammonium fluoride $(500 \mu \mathrm{L}$, 
$0.5 \mathrm{mmol}, 10$ equiv) was added. The solution was stirred for $15 \mathrm{~min}$ at $\mathrm{rt}$ when found complete by TLC (8:2 ethyl acetate:hexanes). Evaporation and purification by flash silica chromatography (8:2 ethyl acetate:hexanes) afforded the alcohol 2 as white solid (13 $\mathrm{mg}, 61 \%)$.

4-Trityloxymethyl-1-(2',3',4',6'-tetra-O-acetyl- $\beta$-D-glucopyranosyl)-1,2,3-triazole (4). To a solution of the alcohol $2(200 \mathrm{mg}, 0.47 \mathrm{mmol})$ dry pyridine $(5 \mathrm{~mL})$ was added trityl chloride (230 mg, $0.84 \mathrm{mmol}, 1.8$ equiv). The solution was warmed to $40{ }^{\circ} \mathrm{C}$ and stirred for $24 \mathrm{~h}$. The solvent was evaporated under reduced pressure and the remaining crude yellow solid was purified by flash silica chromatography (1:9 ethyl acetate:petroleum ether) to afford a crude white solid. A final recrystallization from hot absolute ethanol to remove trace trityl alcohol afforded a white crystalline solid (283 mg, 90\%). Mp: 192-193 ${ }^{\circ} \mathrm{C} . R_{f}=0.63$ (7:3 ethyl acetate:hexanes). ${ }^{1} \mathrm{H}$ NMR (400 MHz, $\left.\mathrm{CDCl}_{3}\right): \delta 1.87\left(\mathrm{~s}, 3 \mathrm{H}, \mathrm{OAc} \mathrm{CH}_{3}\right) 2.02\left(\mathrm{~s}, 3 \mathrm{H}, \mathrm{OAc} \mathrm{CH}_{3}\right) 2.06\left(\mathrm{~s}, 3 \mathrm{H}, \mathrm{OAc}_{3} \mathrm{CH}_{3}\right) 2.09$ $(\mathrm{s}, 3 \mathrm{H}, \mathrm{OAc} \mathrm{CH}) 4.00\left(\mathrm{ddd},{ }^{3} \mathrm{~J}_{5-4}=10 \mathrm{~Hz},{ }^{3} \mathrm{~J}_{5-6},=4.8 \mathrm{~Hz},{ }^{3} \mathrm{~J}_{5-6}=2 \mathrm{~Hz}, 1 \mathrm{H}, \mathrm{H}-5\right) 4.16\left(\mathrm{dd},{ }^{2} \mathrm{~J}_{6-6},=\right.$ $\left.12.8 \mathrm{~Hz},{ }^{3} \mathrm{~J}_{6-5}=2.4 \mathrm{~Hz}, 1 \mathrm{H}, \mathrm{H}-6\right) 4.28\left(\mathrm{~s}, 2 \mathrm{H}, \mathrm{CH}_{2} \mathrm{OTr}\right) 4.31\left(\mathrm{dd},{ }^{2} \mathrm{~J}_{6^{\prime}-6}=12.8 \mathrm{~Hz},{ }^{3} \mathrm{~J}_{6^{\prime}-5}=5.2 \mathrm{~Hz}\right.$, 1H, H-6') 5.23 - 5.28 (m, 1H, H-4) 5.39 - 5.44 (m, 1H, H-3) 5.47 - $5.51(\mathrm{~m}, 1 \mathrm{H}, \mathrm{H}-2) 5.87$ (d, $\left.{ }^{3} \mathrm{~J}_{1-2}=9.6 \mathrm{~Hz}, 1 \mathrm{H}, \mathrm{H}-1\right) 7.22-7.32(\mathrm{~m}, 10 \mathrm{H}, \mathrm{Ar} \mathrm{H}) 7.46-7.49(\mathrm{~m}, 5 \mathrm{H}, \mathrm{Ar} \mathrm{H}) 7.75(\mathrm{~s}, 1 \mathrm{H}$, triazole $\mathrm{CH}$ ); ${ }^{13} \mathrm{C}$ NMR $\left(100 \mathrm{MHz}, \mathrm{CDCl}_{3}\right): \delta 20.4$ (OAc) 20.76 (OAc) 20.77 (OAc) 20.9 (OAc) 58.8 (C-5) 61.8 (C-6) 68.0 (C-4) $70.4(\mathrm{C}-2) 73.0(\mathrm{C}-3) 75.4\left(\mathrm{CH}_{2} \mathrm{OTr}\right) 85.9(\mathrm{C}-1) 87.6\left(\mathrm{CPh}_{3}\right)$ $120.5($ triazole $\mathrm{CH}) 127.4(\mathrm{Ar} \mathrm{CH}) 128.2(\mathrm{Ar} \mathrm{CH}) 128.9(\mathrm{Ar} \mathrm{CH}) 143.8($ triazole $\mathrm{C}) 147.1(\mathrm{Ar} \mathrm{C})$ $169.1(\mathrm{OAc}) 169.6$ (OAc) $170.2(\mathrm{OAc}) 170.7$ (OAc). HRESIMS calculated for $\mathrm{C}_{36} \mathrm{H}_{37} \mathrm{~N}_{3} \mathrm{O}_{10} \mathrm{Na}^{+}$: 694.237116. Found 694.237058. Anal. Calculated for $\mathrm{C}_{36} \mathrm{H}_{37} \mathrm{~N}_{3} \mathrm{O}_{10}$ : C, 64.37; H, 5.55; N, 6.26; O, 23.82. Found: C, 64.12; H, 5.38; N, 6.35. 
Deprotection of trityl ether $4 \rightarrow \mathbf{2}$. A portion of the trityl ether 4 (42 $\mathrm{mg}, 0.06 \mathrm{mmol}$ ) was dissolved in 1:1 $\mathrm{CH}_{2} \mathrm{Cl}_{2}$ :methanol $(3 \mathrm{~mL})$. A catalytic amount of camphorsulfonic acid was then added and the solution was stirred vigorously at $40{ }^{\circ} \mathrm{C}$ for $1 \mathrm{~h}$, at which time TLC indicated reaction completion (7:3 ethyl acetate:hexanes). The solution was neutralized by the addition of $\mathrm{Et}_{3} \mathrm{~N}(10 \mu \mathrm{L}, 0.07 \mathrm{mmol})$ and extracted twice with $\mathrm{CH}_{2} \mathrm{Cl}_{2}(2 \times 2 \mathrm{~mL})$. The combined organic extracts were washed with distilled $\mathrm{H}_{2} \mathrm{O}(4 \mathrm{~mL})$ and the organic layer was dried $\left(\mathrm{MgSO}_{4}\right)$, filtered and evaporated. The resulting crude residue was purified by gradient flash silica chromatography (2:8 ethyl acetate:hexanes to neat ethyl acetate gradient) to afford the alcohol $\mathbf{2}$ as white solid (25 $\mathrm{mg}, 95 \%)$.

4-Acetoxymethyl-1-(2',3',4',6'-tetra-O-acetyl- $\beta$-D-glucopyranosyl)-1,2,3-triazole (5). The alcohol 2 (200 mg, $0.47 \mathrm{mmol})$ was dissolved in dry pyridine $(2 \mathrm{~mL})$ and acetic anhydride $(1 \mathrm{~mL})$ and a catalytic amount of DMAP was added. The solution was stirred at $\mathrm{rt}$ for $1 \mathrm{~h}$ when TLC indicated reaction completion (1:1 ethyl acetate:hexanes). Removal of the solvent under reduced pressure afforded a crude off-white solid which was recrystallized from hot absolute ethanol to afford the peracetate 5 as an off-white amorphous solid $(185 \mathrm{mg}, 84 \%)$. Mp: $143-144{ }^{\circ} \mathrm{C} \cdot R_{f}=$ 0.48 (7:3 ethyl acetate:hexanes). ${ }^{1} \mathrm{H}$ NMR (400 MHz, $\left.\mathrm{CDCl}_{3}\right): \delta 1.86(\mathrm{~s}, 3 \mathrm{H}, \mathrm{OAc}) 2.01(\mathrm{~s}, 3 \mathrm{H}$, OAc) $2.05(\mathrm{~s}, 3 \mathrm{H}, \mathrm{OAc}) 2.07(\mathrm{~s}, 3 \mathrm{H}, \mathrm{OAc}) 2.08(\mathrm{~s}, 3 \mathrm{H}, \mathrm{OAc}) 3.98\left(\mathrm{ddd},{ }^{3} \mathrm{~J}_{5-4}=10.0 \mathrm{~Hz},{ }^{3} \mathrm{~J}_{5-6}=\right.$ $\left.4.8 \mathrm{~Hz},{ }^{3} \mathrm{~J}_{5-6^{\prime}}=2 \mathrm{~Hz}, 1 \mathrm{H}, \mathrm{H}-5\right) 4.14\left(\mathrm{dd},{ }^{2} \mathrm{~J}_{6^{\prime}-6}=12.8 \mathrm{~Hz},{ }^{3} \mathrm{~J}_{6^{\prime}-5}=2 \mathrm{~Hz}, 1 \mathrm{H}, \mathrm{H}-6\right.$ ') 4.29 (dd, ${ }^{2} \mathrm{~J}_{6-6^{\prime}}=$ $\left.12.8 \mathrm{~Hz}, 3 \mathrm{~J}_{6-5}=5.2 \mathrm{~Hz}, 1 \mathrm{H}, \mathrm{H}-6\right) 5.20\left(\mathrm{~s}, 2 \mathrm{H}, \mathrm{CH}_{2} \mathrm{OAc}\right) 5.21-5.24(\mathrm{~m}, 1 \mathrm{H}, \mathrm{H}-4) 5.39-5.41(\mathrm{~m}$, 2H, H-2, H-3) $5.84-5.86(\mathrm{~m}, 1 \mathrm{H}, \mathrm{H}-1) 7.82(\mathrm{~s}, 1 \mathrm{H}$, triazole $\mathrm{CH}) ;{ }^{13} \mathrm{C}$ NMR $\left(100 \mathrm{MHz}, \mathrm{CDCl}_{3}\right)$ : $\delta 20.3$ (OAc) 20.69 (OAc) 20.72 (OAc) 20.9 (OAc) 21.0 (OAc) 57.6 (C-5) 61.7 (C-6) 67.9 (C-4) $70.5\left(\mathrm{CH}_{2} \mathrm{OAc}\right) 72.8(\mathrm{C}-3) 75.4(\mathrm{C}-2) 86.0(\mathrm{C}-1) 122.3$ (triazole $\left.\mathrm{CH}\right) 143.9$ (triazole $\left.\mathrm{C}\right) 169.1$ 
(OAc) 169.5 (OAc) 170.1 (OAc) 170.7 (OAc) 171.0 (OAc). HRESIMS calculated for $\mathrm{C}_{19} \mathrm{H}_{25} \mathrm{~N}_{3} \mathrm{O}_{11} \mathrm{Na}^{+}$: 494.13810. Found: 494.137458. Anal. Calculated for $\mathrm{C}_{19} \mathrm{H}_{25} \mathrm{~N}_{3} \mathrm{O}_{11}$ : C, 54.03; H, 5.10; N, 7.88. Found: C, 53.91; H, 5.12; N, 7.74.

Deprotection of peracetate $5 \rightarrow \mathbf{1 1}$. A portion of the peracetate $(100 \mathrm{mg}$ ) was suspended in dry methanol $(3 \mathrm{~mL})$ and a $1.0 \mathrm{M}$ solution of sodium methoxide in dry methanol $(30 \mu \mathrm{L}, 0.03 \mathrm{mmol})$ was added. The resulting pale yellow solution was stirred at $\mathrm{rt}$ for $15 \mathrm{~min}$ before reaction completion. The solution was neutralized by the addition of dry Amberlite IR-120 acidic ion exchange resin, filtered and evaporated to dryness under reduced pressure to afford the pentol 11 as white solid (53 mg, $96 \%$ ). See compound $\mathbf{1 1}$ for analytical details.

4-Benzoyloxymethyl-1-(2',3',4',6'-tetra-O-acetyl- $\beta$-D-glucopyranosyl)-1,2,3-triazole (6). To a solution of the alcohol $2(200 \mathrm{mg}, 0.47 \mathrm{mmol})$ in dry $\mathrm{CH}_{2} \mathrm{Cl}_{2}(5 \mathrm{ml})$ and $\mathrm{Et}_{3} \mathrm{~N}(195 \mu \mathrm{L}, 1.41$ mmol, 3 equiv) was added benzoyl chloride $(52 \mu \mathrm{L}, 0.71 \mathrm{mmol}, 1.5$ equiv) dropwise. The reaction stirred at $\mathrm{rt}$ for $2 \mathrm{~h}$ when found complete by TLC. $\mathrm{CH}_{2} \mathrm{Cl}_{2}(5 \mathrm{~mL})$ was then added and the organic layer washed consecutively with $1 N \mathrm{HCl}(2 \times 5 \mathrm{~mL})$, saturated $\mathrm{NaHCO}_{3}(2 \times 5 \mathrm{~mL})$ and brine $(5 \mathrm{~mL})$. The organic layer was dried $\left(\mathrm{MgSO}_{4}\right)$, filtered and evaporated to afford crude, off-white solid which was recrystallized from hot absolute ethanol to afford the benzoyl ester $\mathbf{5}$ as an off-white, amorphous solid (220 mg, 88\%). Mp: 180-181 ${ }^{\circ} \mathrm{C} . R_{f}=0.53$ (7:3 ethyl acetate:hexanes). ${ }^{1} \mathrm{H}$ NMR $\left(\mathrm{CDCl}_{3}, 400 \mathrm{MHz}\right): \delta 1.82$ (s, 3H, OAc) 2.00 (s, 3H, OAc) 2.04 (s, $3 \mathrm{H}, \mathrm{OAc}) 2.06(\mathrm{~s}, 3 \mathrm{H}, \mathrm{OAc}) 3.99\left(\mathrm{ddd},{ }^{3} \mathrm{~J}_{5-4},=10.2 \mathrm{~Hz},{ }^{3} \mathrm{~J}_{5-6}=5.2 \mathrm{~Hz},{ }^{3} \mathrm{~J}_{5-6},=2 \mathrm{~Hz}, 1 \mathrm{H}, \mathrm{H}-5\right)$ $4.13\left(\mathrm{dd},{ }^{2} \mathrm{~J}_{6-6^{\prime}}=12.8 \mathrm{~Hz},{ }^{3} \mathrm{~J}_{6^{\prime}-5}=2.0 \mathrm{~Hz}, 1 \mathrm{H}, \mathrm{H}-6^{\prime}\right) 4.28\left(\mathrm{dd},{ }^{2} \mathrm{H}_{6-6^{\prime}}=12.8 \mathrm{~Hz},{ }^{3} \mathrm{~J}_{6-5}=4.8 \mathrm{~Hz}, 1 \mathrm{H}\right.$, H-6) $5.20-5.25(\mathrm{~m}, 1 \mathrm{H}, \mathrm{H}-4) 5.37-5.41(\mathrm{~m}, 1 \mathrm{H}, \mathrm{H}-3) 5.41-5.50\left(\mathrm{~m}, 3 \mathrm{H}, \mathrm{H}-2, \mathrm{CH}_{2} \mathrm{OBz}\right) 5.87$ 
$\left(\mathrm{d},{ }^{3} \mathrm{~J}_{1-2}=9.2 \mathrm{~Hz}, 1 \mathrm{H}, \mathrm{H}-1\right) 7.39(\mathrm{~m}, 2 \mathrm{H}, \mathrm{Ar} \mathrm{H}) 7.52(\mathrm{~m}, 1 \mathrm{H}, \mathrm{Ar} \mathrm{H}) 7.91$ (s, 1H, triazole H) $8.02-$

$8.04(\mathrm{~m}, 2 \mathrm{H}, \mathrm{Ar} \mathrm{H}),{ }^{13} \mathrm{C}$ NMR (100 MHz, $\mathrm{CDCl}_{3}$ ): $\delta 20.3$ (OAc) 20.70 (OAc) 20.73 (OAc) 20.9

(OAc) 58.1 (C-5) 61.7 (C-6) 67.9 (C-4) $70.5\left(\mathrm{CH}_{2} \mathrm{OBz}\right) 72.8$ (C-3) 75.4 (C-2) 86.0 (C-1) 122.6 (triazole $\mathrm{CH}) 128.6(\mathrm{Ar} \mathrm{CH}) 129.9(\mathrm{Ar} \mathrm{CH}) 130.0(\mathrm{Ar} \mathrm{CH}) 133.5$ (Ar C) 143.9 (triazole C) 166.5 $(\mathrm{OBz} C=\mathrm{O}) 169.0(\mathrm{OAc} \mathrm{C}=\mathrm{O}) 169.5(\mathrm{OAc} \mathrm{C}=\mathrm{O}) 170.1 \quad(\mathrm{OAc} \mathrm{C}=\mathrm{O}) 170.7(\mathrm{OAc} \mathrm{C}=\mathrm{O})$. HRESIMS calculated for $\mathrm{C}_{24} \mathrm{H}_{27} \mathrm{~N}_{3} \mathrm{O}_{11} \mathrm{Na}^{+}$: 556.15378. Found: 556.153103. Anal. Calculated for $\mathrm{C}_{24} \mathrm{H}_{27} \mathrm{~N}_{3} \mathrm{O}_{11}:$ C, 54.03; H, 5.10; N, 7.88. Found: C, 53.91; H, 5.12; N, 7.74.

Deprotection of benzoyl ester $6 \rightarrow \mathbf{1 1}$. A portion of the benzoyl ester $6(25 \mathrm{mg}, 0.047 \mathrm{mmol})$ was suspended in dry methanol $(2 \mathrm{~mL})$ and a $1.0 \mathrm{M}$ solution of sodium methoxide $(10 \mu \mathrm{L}, 0.01$ mmol) in dry methanol was added. The pale yellow solution was stirred $\mathrm{o} / \mathrm{n}$ at $40{ }^{\circ} \mathrm{C}$ to effect complete benzoyl deprotection. TLC indicated reaction completion after $12 \mathrm{~h}$ (8:2 ethyl acetate:hexanes and 1:9 water:acetonitrile) and the solution was neutralized by the addition of Amberlite IR-120 acidic ion-exchange resin. Filtration and evaporation to dryness under reduced pressure afforded the pentol $\mathbf{1 1}$ as white solid (10 $\mathrm{mg}, 82 \%)$.

4-Benzyloxymethyl-1-(2',3',4',6'-tetra-O-benzyl- $\beta$-D-glucopyranosyl)-1,2,3-triazole (7). To a solution of the pentol $11(140 \mathrm{mg}, 0.54 \mathrm{mmol})$ in dry DMF $(5 \mathrm{~mL})$ under nitrogen was added at 0 ${ }^{\circ} \mathrm{C}$ a $60 \%$ sodium hydride mineral oil dispersion $(130 \mathrm{mg}, 3.24 \mathrm{mmol}, 6$ equiv). The heterogenous mixture was allowed to warm to room temperature and stirred for an additional 30 min before benzyl bromide (380 $\mu \mathrm{L}, 3.24 \mathrm{mmol}, 6$ equiv) was added. The deep yellow solution was stirred at rt for $8 \mathrm{~h}$. The solution was then cooled again to $0{ }^{\circ} \mathrm{C}$ and methanol was added gradually. Bulk solvent was then removed and $\mathrm{CH}_{2} \mathrm{Cl}_{2}(20 \mathrm{~mL})$ was added. The organic extract was washed 
consecutively with $1 N \mathrm{HCl}(2 \times 5 \mathrm{~mL})$, saturated $\mathrm{NaHCO}_{3}(2 \times 5 \mathrm{~mL})$ and brine $(5 \mathrm{~mL})$. The organic layer was dried $\left(\mathrm{MgSO}_{4}\right)$, filtered and evaporated under reduced pressure to afford a pale yellow oil which was purified by flash silica chromatography (2:8 ethyl acetate:hexanes) to afford the pentabenzyl ether 7 as white crystalline solid $(330 \mathrm{mg}, 86 \%) . \mathrm{Mp}=98-99{ }^{\circ} \mathrm{C} . R_{f}=$ 0.43 (6:4 ethyl acetate:hexanes). ${ }^{1} \mathrm{H}$ NMR (400 MHz, $\left.\mathrm{CDCl}_{3}\right): \delta 3.65-3.76(\mathrm{~m}, 3 \mathrm{H}, \mathrm{H}-5, \mathrm{H}-6$, H-6') $3.78-3.89$ (m, 2H, H-3, H-4) $4.02-4.06$ (m, 2H, H-2) $4.07-4.09$ (m, 2H, CH $4.47-4.56\left(\mathrm{~m}, 4 \mathrm{H}, \mathrm{CH}_{2} \mathrm{Ph}\right) 4.59-4.67\left(\mathrm{~m}, 2 \mathrm{H}, \mathrm{CH}_{2} \mathrm{Ph}\right) 4.67-4.73\left(\mathrm{~m}, 2 \mathrm{H}, \mathrm{CH}_{2} \mathrm{Ph}\right) 4.88-4.95$ $\left(\mathrm{m}, 2 \mathrm{H}, \mathrm{CH}_{2} \mathrm{Ph}\right) 5.96\left(\mathrm{~d},{ }^{3} \mathrm{~J}_{1-2}=9.6 \mathrm{~Hz}, 1 \mathrm{H}, \mathrm{H}-1\right) 6.93-6.97(\mathrm{~m}, 2 \mathrm{H}, \mathrm{Ar} \mathrm{CH}) 7.16-7.19(\mathrm{~m}, 4 \mathrm{H}$, Ar CH) $7.27-7.34(\mathrm{~m}, 19 \mathrm{H}, \mathrm{Ar} \mathrm{CH}) 7.66(\mathrm{~s}, 1 \mathrm{H}$, triazole $\mathrm{CH}) ;{ }^{13} \mathrm{C}$ NMR $\left(100 \mathrm{MHz}, \mathrm{CDCl}_{3}\right)$ : $63.8\left(\mathrm{CH}_{2} \mathrm{Ph}\right) 68.6(\mathrm{C}-6) 72.2\left(\mathrm{CH}_{2} \mathrm{Ph}\right) 73.8\left(\mathrm{CH}_{2} \mathrm{Ph}\right) 75.1\left(\mathrm{CH}_{2} \mathrm{OBn}\right) 75.4\left(\mathrm{CH}_{2} \mathrm{Ph}\right) 76.0\left(\mathrm{CH}_{2} \mathrm{Ph}\right)$ 77.5 (C-3 or C-4) 78.2 (C-5) 81.0 (C-2) 85.7 (C-3 or C-4) 87.8 (C-1) 122.2 (triazole CH) 127.94 (Ar C) $127.99(\operatorname{Ar~C}) 128.01(\operatorname{Ar~C}) 128.03(\operatorname{Ar~C}) 128.06(\operatorname{Ar~C}) 128.09(\operatorname{Ar~C}) 128.17(\operatorname{Ar~C})$ $128.18(\mathrm{Ar} \mathrm{C}) 128.23(\mathrm{Ar} \mathrm{C}) 128.46(\mathrm{Ar} \mathrm{C}) 128.59(\mathrm{Ar} \mathrm{C}) 128.64(\mathrm{Ar} \mathrm{C}) 128.67$ ( $\mathrm{Ar} \mathrm{C}) 128.72$ (Ar C) 137.2 (Ar C) 137.9 (Ar C) 138.0 (2 x Ar C) 138.4 (Ar C) 145.7 (triazole C). HRESIMS: calculated for $\mathrm{C}_{44} \mathrm{H}_{45} \mathrm{~N}_{3} \mathrm{O}_{6} \mathrm{Na}^{+}=734.320057$, found $=734.320207$. Anal. Calculated for $\mathrm{C}_{44} \mathrm{H}_{45} \mathrm{~N}_{3} \mathrm{O}_{6}$ : C, 74.24; H, 6.37; N, 5.90; O, 13.49. Found: C, 74.64; H, 6.49; N, 5.71.

Deprotection of perbenzyl ether $7 \rightarrow$ 11. The perbenzyl ether $6(20 \mathrm{mg}, 0.03 \mathrm{mmol})$ was dissolved dry 1:2 $\mathrm{CH}_{2} \mathrm{Cl}_{2}$ :methanol $(\sim 2 \mathrm{~mL})$ and a catalytic amount of activated $30 \% \mathrm{Pd}(\mathrm{OH})_{2} / \mathrm{C}$ was added. The vessel was placed under nitrogen, evacuated and then placed under an atmosphere of hydrogen and stirred at $\mathrm{rt}$ for $24 \mathrm{~h}$. TLC indicated near reaction completion after ca. $24 \mathrm{~h}$ (8:2 ethyl acetate:hexanes and 1:9 water:acetonitrile). The mixture was filtered on celite 
and eluted several times with methanol. Evaporation of the filtrate afforded the pentol $\mathbf{1 1}$ as clear gum (6 mg, 78\%).

[1-(2,3,4,6-tetra-O-acetylglucpyranosyl)-1H-1,2,3-triazol-4-yl]methyl $\quad 2,3,4,6$-tetra-O-acetyl glucopyranoside (8). A solution of the alcohol 2 (100 mg, $0.23 \mathrm{mmol})$ and 2,3,4,6-tetra-Oacetyl- $\alpha$-D-glucosyl bromide (172 mg, $0.42 \mathrm{mmol}, 1.8$ equiv) in dry $\mathrm{CH}_{2} \mathrm{Cl}_{2}(5 \mathrm{~mL})$ was prepared under nitrogen. Silver trifluoromethansulfonate $(66 \mathrm{mg}, 0.26 \mathrm{mmol}, 1.1$ equiv) was then added and the mixture stirred at $\mathrm{rt}$ for $4 \mathrm{~h}$ when found complete by TLC (7:3 ethyl acetate:hexanes). The solution was neutralized by the addition of diisopropylethylamine (100 $\mu \mathrm{L}, 0.57 \mathrm{mmol})$. Insoluble silver salts were removed by filtration on celite and the crude product was eluted with $\mathrm{CH}_{2} \mathrm{Cl}_{2}(\sim 20 \mathrm{~mL})$. Concentration of the filtrate under reduced pressure and flash silica chromatography of the crude residue (4:6 ethyl acetate:hexanes) afforded the glucoside $\mathbf{8}$ as a white foam (98 mg, 56\%). ${ }^{1} \mathrm{H}$ NMR (400 MHz, $\left.\mathrm{CDCl}_{3}\right): \delta 1.88$ (s, 3H, OAc) 1.95 (s, 3H, OAc) $1.97(\mathrm{~s}, 3 \mathrm{H}, \mathrm{OAc}) 2.00(\mathrm{~s}, 3 \mathrm{H}, \mathrm{OAc}) 2.02(\mathrm{~s}, 3 \mathrm{H}, \mathrm{OAc}) 2.05(\mathrm{~s}, 3 \mathrm{H}, \mathrm{OAc}) 2.06(\mathrm{~s}, 3 \mathrm{H}, \mathrm{OAc}) 2.10$ (s, 3H, OAc) $3.74\left(\mathrm{ddd}, 1 \mathrm{H}, 3 \mathrm{~J}_{5-4}=10.2 \mathrm{~Hz},{ }^{3} \mathrm{~J}_{5-6}=4.4 \mathrm{~Hz}^{3} \mathrm{~J}_{5-6},=2.4 \mathrm{~Hz}, 1 \mathrm{H}, \mathrm{GlcO} \mathrm{H}-5\right) ; 3.99$ $\left(\mathrm{ddd}, 3 \mathrm{~J}_{5-4}=10.4 \mathrm{~Hz}, 3 \mathrm{~J}_{5-6}=4.8 \mathrm{~Hz}, 3 \mathrm{~J}_{5-6}{ }^{\prime}=2 \mathrm{~Hz}, 1 \mathrm{H}, \mathrm{GlcN} \mathrm{H-5}\right), 4.13\left(\mathrm{dd},{ }^{2} \mathrm{~J}_{6}{ }^{\prime}-6=12.4 \mathrm{~Hz},{ }^{3} \mathrm{~J}_{6}{ }^{\prime}\right.$ $\left.{ }_{5}=2.0 \mathrm{~Hz}, 1 \mathrm{H}, \mathrm{GlcN} H-6^{\prime}\right) 4.18\left(\mathrm{dd},{ }^{2} \mathrm{~J}_{6}{ }^{\prime}-6=12.4 \mathrm{~Hz},{ }^{3} \mathrm{~J}_{6}{ }^{\prime}-5=2.4 \mathrm{~Hz}, 1 \mathrm{H}\right.$, GlcO H-6') $4.26-4.32$ (m, 2H, GlcN H-6, GlcO H-6) $4.54\left(\mathrm{~d},{ }^{3} \mathrm{~J}_{1-2}=8 \mathrm{~Hz}, 1 \mathrm{H}, \mathrm{GlcO} \mathrm{H}-1\right) 4.78-4.92\left(\mathrm{~m}, 2 \mathrm{H}, \mathrm{CH}_{2} \mathrm{O}\right)$ $4.98\left(\mathrm{dd},{ }^{3} \mathrm{~J}_{2-3}=9.6 \mathrm{~Hz},{ }^{3} \mathrm{~J}_{2-1}=8.4 \mathrm{~Hz}, 1 \mathrm{H}, \mathrm{GlcO} \mathrm{H}-2\right) 5.06-5.11(\mathrm{~m}, 1 \mathrm{H}, \mathrm{GlcO} \mathrm{H}-4) 5.15-5.20$ (m, 1H, GlcO H-3) 5.21 - $5.26(\mathrm{~m}, 1 \mathrm{H}, \mathrm{GlcN}$ H-4) 5.39 - 5.42 (m, 2H, GlcN H-2, H-3) 5.84 $5.86(\mathrm{~m}, 1 \mathrm{H}, \mathrm{GlcN} \mathrm{H}-1) 7.80(\mathrm{~s}, 1 \mathrm{H}$, triazole $\mathrm{H}) ;{ }^{13} \mathrm{C} \mathrm{NMR}\left(100 \mathrm{MHz}, \mathrm{CDCl}_{3}\right): \delta 20.3(\mathrm{OAc})$ $20.72(2$ x OAc) $20.79(3$ x OAc) 20.86 (OAc) 20.89 (OAc) 61.7 (GlcN or GlcO C-6) 62.0 (GlcN or GlcO C-6) $62.1\left(\mathrm{CH}_{2} \mathrm{O}\right) 67.9$ (GlcN C-4) 68.6 (GlcO C-4) 70.6 (GlcN C-2 or C-3) 71.3 (GlcO 
C-2) 72.0 (GlcO C-5) 72.6 (GlcN C-2 or C-3) 72.8 (GlcO C-3) 75.5 (GlcN C-5) 86.1 (GlcN C-1) 99.0 (GlcO C-1) 122.3 (triazole $\mathrm{CH}) 169.55$ (OAc) 169.57 (OAc) 169.62 (OAc) 170.05 (OAc) 170.1 (OAc) 170.4 (OAc) 170.6 (OAc) 170.9 (OAc). HRESIMS calculated for $\mathrm{C}_{31} \mathrm{H}_{41} \mathrm{~N}_{3} \mathrm{O}_{19} \mathrm{Na}^{+}$: 782.22267. Found: 782.223136. Anal. Calculated for $\mathrm{C}_{31} \mathrm{H}_{41} \mathrm{~N}_{3} \mathrm{O}_{19}$ : C, 49.01; H, 5.44; N, 5.53; O, 40.02. Found: C, 48.93; H, 5.61; N, 5.17.

\section{[1-(2,3,4,6-tetra-O-acetylglucpyranosyl)-1H-1,2,3-triazol-4-yl]methyl $\quad 2,3,4,6$-tetra-O-acetyl}

galactopyranoside (9). A solution of the alcohol $2(157 \mathrm{mg}, 0.37 \mathrm{mmol})$ and $\beta$-D-galactose pentaacetate (143 mg, $0.37 \mathrm{mmol}, 1$ equiv) in dry $\mathrm{CH}_{2} \mathrm{Cl}_{2}(5 \mathrm{~mL})$ was prepared under nitrogen and cooled to $0{ }^{\circ} \mathrm{C}$. Boron trifluoride etherate $(113 \mu \mathrm{L}, 0.92 \mathrm{mmol}, 2.5$ equiv) was then added dropwise and the reaction was allowed to warm to rt. The reaction was found complete by TLC (8:2 ethyl acetate:hexanes) after stirring under nitrogen o/n. $\mathrm{CH}_{2} \mathrm{Cl}_{2}(5 \mathrm{ml})$ was added and washed with saturated $\mathrm{NaHCO}_{3}(2 \times 5 \mathrm{~mL})$ and brine $(5 \mathrm{~mL})$. The organic layer was dried $\left(\mathrm{MgSO}_{4}\right)$, filtered and evaporated to a pale yellow oil which was purified by flash silica chromatography to afford the galactoside $\mathbf{1 0}$ as a clear gum which slowly crystallized on standing in the refrigerator (165 mg, 59\%). Mp: $106-107^{\circ} \mathrm{C} .{ }^{1} \mathrm{H}$ NMR (400 MHz, $\left.\mathrm{CDCl}_{3}\right): \delta 1.85$ (s, 3H OAc) $1.92(\mathrm{~s}, 3 \mathrm{H}$ OAc) $1.93(\mathrm{~s}, 3 \mathrm{H}, \mathrm{OAc}) 2.00(\mathrm{~s}, 3 \mathrm{H}, \mathrm{OAc}) 2.03(\mathrm{~s}, 3 \mathrm{H}, \mathrm{OAc}) 2.04(\mathrm{~s}, 3 \mathrm{H}, \mathrm{OAc}) 2.05(\mathrm{~s}, 3 \mathrm{H}$, OAc) $2.12(\mathrm{~s}, 3 \mathrm{H}, \mathrm{OAc}) 3.92-3.96(\mathrm{~m}, 1 \mathrm{H}, \mathrm{Gal} \mathrm{H}-5) 4.00\left(\mathrm{ddd},{ }^{3} \mathrm{~J}_{5-4}=7.2 \mathrm{~Hz},{ }^{3} \mathrm{~J}_{5-6}=5.2 \mathrm{~Hz},{ }^{3} \mathrm{~J}_{5-}\right.$ $\left.6^{\prime}=2 \mathrm{~Hz}, 1 \mathrm{H}, \mathrm{Glc} \mathrm{H}-5\right) 4.07-4.13\left(\mathrm{~m}, 2 \mathrm{H}\right.$, Glc H-6', Gal H-6') $4.21\left(\mathrm{dd},{ }^{2} \mathrm{~J}_{6-6^{\prime}}=11.6 \mathrm{~Hz},{ }^{3} \mathrm{~J}_{6-5}=\right.$ $6.4 \mathrm{~Hz}, 1 \mathrm{H}$, Gal H-6) $4.27\left(\mathrm{dd},{ }^{2} \mathrm{~J}_{6-6},=12.8 \mathrm{~Hz},{ }^{3} \mathrm{~J}_{6-5}=5.2 \mathrm{~Hz}, 1 \mathrm{H}, \mathrm{Glc} \mathrm{H}-6\right) 4.46\left(\mathrm{~d},{ }^{3} \mathrm{~J}_{1-2}=8 \mathrm{~Hz}\right.$, 1H, Gal H-1) $4.76-4.92\left(\mathrm{~m}, 2 \mathrm{H}, \mathrm{CH}_{2} \mathrm{O}\right) 5.17\left(\mathrm{dd},{ }^{3} \mathrm{~J}_{2-3}=10.8 \mathrm{~Hz},{ }^{3} \mathrm{~J}_{2-1}=8 \mathrm{~Hz}, 1 \mathrm{H}, \mathrm{Gal} \mathrm{H}-2\right) 4.98$ $\left.\left(\mathrm{dd},{ }^{3} \mathrm{~J}_{3-2}=10.4 \mathrm{~Hz},{ }^{3} \mathrm{~J}_{3-4}=3.2 \mathrm{~Hz}, 1 \mathrm{H}, \mathrm{Gal} \mathrm{H}-3\right)\right) 5.21-5.26(\mathrm{~m}, 1 \mathrm{H}, \mathrm{Glc} \mathrm{H}-4) 5.35-5.43(\mathrm{~m}$, $3 \mathrm{H}$, Glc H-2, Glc H-3, Gal H-4) $5.85\left(\mathrm{~d},{ }^{3} \mathrm{~J}_{1-2}=8.8 \mathrm{~Hz}, 1 \mathrm{H}\right.$, Glc H-1) $7.80(\mathrm{~s}, 1 \mathrm{H}$, triazole $\mathrm{H}) ;{ }^{13} \mathrm{C}$ 
NMR (100 MHz, CDCl $): \delta 20.3$ (OAc) 20.69 (OAc) 20.71 (OAc) 20.75 (OAc) 20.83 (2 x OAc) $20.87(\mathrm{OAc}) 20.9(\mathrm{OAc}) 61.5\left(\mathrm{CH}_{2} \mathrm{O}\right) 61.6(\mathrm{Gal} \mathrm{C}-6) 61.7$ (Glc C-6) 67.3 (Gal C-4) 67.9 (Gal C2) 68.8 (Glc C-4) 70.7 (Gal C-5) 70.8 (Glc C-3) 71.0 (Gal C-3) 72.6 (Glc C-2) 75.4 (Glc C-5) 86.0 (Glc C-1) 99.1 (Gal C-1) 122.2 (triazole CH) 144.3 (triazole C) 169.3 (OAc) 169.6 (OAc) 169.7 (OAc) $170.0(\mathrm{OAc}) 170.2(\mathrm{OAc}) 170.5$ (OAc) 170.6 (OAc) 170.7 (OAc). HRESIMS: Calculated for $\mathrm{C}_{31} \mathrm{H}_{41} \mathrm{~N}_{3} \mathrm{O}_{19} \mathrm{Na}^{+}$: 782.222647. Found: 782.223784. Anal. Calculated for $\mathrm{C}_{31} \mathrm{H}_{43} \mathrm{~N}_{3} \mathrm{O}_{20}$ (monohydrate): C, 47.88; H, 5.57; N, 5.40. Found: C, 47.62; H, 5.26; N, 5.07.

4-Azidomethyl-1-(2',3',4',6'-tetra-O-acetyl- $\beta$-D-glucopyranosyl)-1,2,3-triazole (10). To a solution of the alcohol $2(200 \mathrm{mg}, 0.47 \mathrm{mmol})$ in dry $\mathrm{CH}_{2} \mathrm{Cl}_{2}(5 \mathrm{~mL})$ was added $\mathrm{Et}_{3} \mathrm{~N}(195 \mu \mathrm{L}$, $1.41 \mathrm{mmol}, 3$ equiv) and methanesulfonyl chloride ( $55 \mu \mathrm{L}, 0.71 \mathrm{mmol}, 1.5$ equiv). The solution was stirred at $\mathrm{rt}$ under nitrogen for $30 \mathrm{~min}$, at which time, TLC indicated reaction completion (7:3 ethyl acetate:hexanes). $\mathrm{CH}_{2} \mathrm{Cl}_{2}(5 \mathrm{~mL})$ was then added and the solution was washed consecutively with $1 N \mathrm{HCl}(2 \times 5 \mathrm{~mL})$, saturated $\mathrm{NaHCO}_{3}(5 \mathrm{~mL})$ and brine $(5 \mathrm{~mL})$. The organic layer was dried $\left(\mathrm{MgSO}_{4}\right)$, filtered and evaporated to afford the mesylate as a pale yellow oil, which was used in the subsequent step without further purification. The crude mesylate was dissolved in dry DMF (2 mL) and $\mathrm{NaN}_{3}(153 \mathrm{mg}, 2.35 \mathrm{mmol}, 5$ equiv) was added. The mixture was warmed to $40{ }^{\circ} \mathrm{C}$ and stirred for $24 \mathrm{~h}$. The bulk solvent was then removed under reduced pressure and $\mathrm{CH}_{2} \mathrm{Cl}_{2}(5 \mathrm{~mL})$ was added. The extract was washed with distilled $\mathrm{H}_{2} \mathrm{O}(2 \mathrm{~mL})$ and brine $(2 \mathrm{~mL})$. The organic layer was dried $\left(\mathrm{MgSO}_{4}\right)$, filtered and evaporated to afford a crude off white solid. Recrystallization from hot absolute ethanol afforded the azide $\mathbf{1 0}$ as amorphous white solid (102 mg, 48\%). Mp: $110-111^{\circ} \mathrm{C} .{ }^{1} \mathrm{H}$ NMR (400 MHz, CDCl $)$ ) 1.87 (s, 3H, OAc) 2.02 (s, $3 \mathrm{H}, \mathrm{OAc}) 2.06(\mathrm{~s}, 3 \mathrm{H}, \mathrm{OAc}) 2.07(\mathrm{~s}, 3 \mathrm{H}, \mathrm{OAc}) 4.00\left(\mathrm{ddd},{ }^{3} \mathrm{~J}_{5-4}=10.4 \mathrm{~Hz},{ }^{3} \mathrm{~J}_{5-6}=5.2 \mathrm{~Hz},{ }^{3} \mathrm{~J}_{5-6},=\right.$ 
$2.4 \mathrm{~Hz}, 1 \mathrm{H}, \mathrm{H}-5) 4.14\left(\mathrm{dd},{ }^{2} \mathrm{~J}_{6-6^{\prime}}=12.8 \mathrm{~Hz},{ }^{3} \mathrm{~J}_{6^{\prime}-5}=2.4 \mathrm{~Hz}, 1 \mathrm{H}, \mathrm{H}-6\right.$ ') $4.30\left(\mathrm{dd},{ }^{2} \mathrm{~J}_{6-6^{\prime}}=12.4 \mathrm{~Hz}\right.$, $\left.{ }^{3} \mathrm{~J}_{6-5}=5.2 \mathrm{~Hz}, 1 \mathrm{H}, \mathrm{H}-6\right) 4.48\left(\mathrm{~s}, 2 \mathrm{H}, \mathrm{CH}_{2} \mathrm{~N}_{3}\right) 5.20-5.25(\mathrm{~m}, 1 \mathrm{H}, \mathrm{H}-4) 5.38-5.44(\mathrm{~m}, 2 \mathrm{H}, \mathrm{H}-2$, H-3) $5.84-5.90(\mathrm{~m}, 1 \mathrm{H}, \mathrm{H}-1) 7.79(\mathrm{~s}, 1 \mathrm{H}$, triazole $\mathrm{CH}) ;{ }^{13} \mathrm{C} \mathrm{NMR}\left(100 \mathrm{MHz}, \mathrm{CDCl}_{3}\right): 20.3$ (OAc) 20.7 (OAc) 20.8 (OAc) 20.9 (OAc) $45.7\left(\mathrm{~N}_{3} \mathrm{CH}_{2}\right) 61.7$ (C-6) 67.9 (C-4) 70.5 (C-3) 72.7 (C-2) 75.5 (C-5) 86.1 (C-1) 121.0 (triazole CH) 169.2 (OAc) 169.6 (OAc) 170.1 (OAc) 170.7 (OAc). HRESIMS: calculated for $\mathrm{C}_{17} \mathrm{H}_{22} \mathrm{~N}_{6} \mathrm{O}_{9} \mathrm{Na}^{+}$: 477.134047. Found 477.134673. Anal. Calculated for $\mathrm{C}_{17} \mathrm{H}_{22} \mathrm{~N}_{6} \mathrm{O}_{9}$ : C, 44.94; H, 4.88; N, 18.50. Found: C, 44.72; H, 4.90; N, 18.27.

1-(ß-D-Glucopyranosyl)-4-hydroxymethyl-1,2,3-triazole (11). White solid (various yields: see text) $\mathrm{Mp}: 162-163{ }^{\circ} \mathrm{C} . R_{f}=0.15$ (1:9 water:acetonitrile). ${ }^{1} \mathrm{H}$ NMR $\left(400 \mathrm{MHz}, \mathrm{D}_{2} \mathrm{O}\right): \delta 3.46-$ 3.50 (m, 1H, ) $3.55-3.67$ (m, 3H, H-3, H-5, H-6) 3.75 - 3.78 (m, 1H, H-6') $3.84-3.89$ (m, 1H, H-2) $4.60\left(\mathrm{~s}, 2 \mathrm{H}, \mathrm{CH}_{2} \mathrm{OH}\right) 5.61\left(\mathrm{~d},{ }^{3} \mathrm{~J}_{1-2}=9.2 \mathrm{~Hz}, 1 \mathrm{H}, \mathrm{H}-1\right) 8.06(\mathrm{~s}, 1 \mathrm{H}$, triazole $\mathrm{CH}) ;{ }^{13} \mathrm{C}$ NMR (100 MHz, D $2 \mathrm{O}): \delta 54.7\left(\mathrm{CH}_{2} \mathrm{OH}\right) 60.6$ (C-6) 69.1 (C-4) 72.4 (C-2) 76.1 (C-3) 79.0 (C-5) 87.6 (C-1) 123.5 (triazole $\mathrm{CH}$ ). HRESIMS calculated for $\mathrm{C}_{9} \mathrm{H}_{15} \mathrm{~N}_{3} \mathrm{O}_{6} \mathrm{Na}^{+}$: 284.085310. Found: 284.085457.

Preparation of compounds 12-18, 20. To a vigorously stirring suspension or solution of the azide $(0.5 \mathrm{M})$ and acetylene $(0.5 \mathrm{M})$ in the selected co-solvent, was added a solution of $\mathrm{CuSO}_{4} .5 \mathrm{H}_{2} \mathrm{O}\left(0.2\right.$ equiv) and sodium ascorbate ( 0.4 equiv) in deionized $\mathrm{H}_{2} \mathrm{O}$. The deep yellow mixture was stirred vigorously at $40^{\circ} \mathrm{C}$ and the reaction progress was monitored by TLC. Once complete, the crude mixture was extracted with 3 equal amounts of $\mathrm{CH}_{2} \mathrm{Cl}_{2}$, combined, dried $\left(\mathrm{MgSO}_{4}\right)$, filtered and evaporated to afford the glycosyl triazole as off-white to white solid. A 
portion or the entire crude solid was purified by SPE, flash chromatography or recrystallization from hot absolute ethanol to afford analytically pure compound.

4-Pyridin-2-yl-1-(2',3',4',6'-tetra-O-acetyl- $\beta$-D-glucopyranosyl)-1,2,3-triazole (12). White amorphous solid (92\%). Mp: $195-196{ }^{\circ} \mathrm{C} . R_{f}=0.42$ (8:2 ethyl acetate:hexane). ${ }^{1} \mathrm{H}$ NMR (400 $\left.\mathrm{MHz}, \mathrm{CDCl}_{3}\right): 1.87$ (s, 3H, OAc) $2.02(\mathrm{~s}, 3 \mathrm{H}, \mathrm{OAc}) 2.06(\mathrm{~s}, 3 \mathrm{H}, \mathrm{OAc}) 2.08(\mathrm{~s}, 3 \mathrm{H}, \mathrm{OAc}) 4.01$ $\left(\mathrm{ddd},{ }^{3} \mathrm{~J}_{5-4}=10.4 \mathrm{~Hz},{ }^{3} \mathrm{~J}_{5-6},=4.8 \mathrm{~Hz},{ }^{3} \mathrm{~J}_{5-6}=2.0 \mathrm{~Hz}, 1 \mathrm{H}, \mathrm{H}-5\right) 4.15\left(\mathrm{dd},{ }^{2} \mathrm{~J}_{6-6},=12.8 \mathrm{~Hz}, \mathrm{~J}_{6-5}=2.0\right.$ $\mathrm{Hz}, 1 \mathrm{H}, \mathrm{H6}) 4.29\left(\mathrm{dd},{ }^{2} \mathrm{~J}_{6^{\prime}-6}=12.4 \mathrm{~Hz},{ }^{3} \mathrm{~J}_{6^{\prime}-5}=4.8 \mathrm{~Hz}, \mathrm{H}-6{ }^{\prime}\right) 5.23-5.28$ (m, 1H, H-4) $5.40-5.45$ (m, 1H, H-3) $5.50-5.55(\mathrm{~m}, 1 \mathrm{H}, \mathrm{H}-2) 5.90\left(\mathrm{~d},{ }^{3} \mathrm{~J}_{1-2}=9.2 \mathrm{~Hz}, 1 \mathrm{H}, \mathrm{H}-1\right) 7.30-7.33(\mathrm{~m}, 1 \mathrm{H}, \mathrm{Ar} \mathrm{H})$ $7.85-7.88\left(\mathrm{~m}, 1 \mathrm{H}\right.$, Ar H) $8.21-8.23(\mathrm{~m}, 1 \mathrm{H}, \mathrm{Ar} \mathrm{H}) 8.65(\mathrm{br} \mathrm{s}, 1 \mathrm{H}$, triazole $\mathrm{H}) ;{ }^{13} \mathrm{C}$ NMR $(100$ $\mathrm{MHz}, \mathrm{CDCl}_{3}$ ): $\delta 20.4$ (OAc) 20.73 (s, 3H, OAc) 20.74 (s, 3H, OAc) 20.9 (s, OAc) 61.7 (C-6) 67.9 (C-4) 70.7 (C-3) 72.9 (C-2) 75.3 (C-5) 86.1 (C-1) 120.7 (triazole CH) 121.0 (Ar C) 123.5 (Ar CH) $137.3(\mathrm{Ar} \mathrm{CH}) 148.9$ (triazole C) 149.5 (Ar CH) 149.6 (Ar C) 169.0 (OAc) 169.5 (OAc) 170.2 (OAc) 170.7 (OAc). HRESIMS: Calculated for $\mathrm{C}_{21} \mathrm{H}_{24} \mathrm{~N}_{4} \mathrm{O}_{9} \mathrm{Na}^{+}$: 499.143549. Found: 499.142640. Anal. Calculated for $\mathrm{C}_{21} \mathrm{H}_{24} \mathrm{~N}_{4} \mathrm{O}_{9}$ : C, 52.44; H, 5.08; N, 11.76; O, 30.22. Found: C, 52.17; H, 5.04; N, 11.36 .

\section{4-Amidomethyl-L-NBocVal-1-(2',3',4',6'-tetra-O-acetyl- $\beta$-D-glucopyranosyl)-1,2,3-triazole}

(13). White foam (77\%). ${ }^{1} \mathrm{H}$ NMR (400 MHz, $\left.\mathrm{CDCl}_{3}\right): \delta 0.85\left(\mathrm{~d},{ }^{3} \mathrm{~J}=6.4 \mathrm{~Hz}, 3 \mathrm{H}\right.$, Val i-pr $\left.\mathrm{CH}_{3}\right)$ $0.91\left(\mathrm{~d},{ }^{3} \mathrm{~J}=7.2 \mathrm{~Hz}\right.$, Val i-pr $\left.\mathrm{CH}_{3}\right) 1.41\left(\mathrm{~s}, 9 \mathrm{H}, \mathrm{Boc} \mathrm{CH}_{3}\right) 1.84$ (s, 3H, OAc) 2.00 (s, 3H, OAc) $2.04(\mathrm{~s}, 3 \mathrm{H}, \mathrm{OAc}) 2.06(\mathrm{~s}, \mathrm{OAc}) 2.13\left(\mathrm{~m}, 1 \mathrm{H}, \mathrm{Val}\right.$ i-pr CH) $3.92(\mathrm{~m}, 1 \mathrm{H}, \mathrm{BocNH}) 3.98\left(\mathrm{ddd},{ }^{3} \mathrm{~J}_{5-4}\right.$ $\left.=10.2 \mathrm{~Hz},{ }^{3} \mathrm{~J}_{5-6}=4.4,{ }^{3} \mathrm{~J}_{5-6},=2.0 \mathrm{~Hz}, 1 \mathrm{H}, \mathrm{H}-5\right) 4.12\left(\mathrm{dd},{ }^{2} \mathrm{~J}_{6-6},=12.8 \mathrm{~Hz},{ }^{3} \mathrm{~J}_{6-5}=2.4 \mathrm{~Hz}, 1 \mathrm{H}, \mathrm{H}-6\right)$ $4.27\left(\mathrm{dd},{ }^{2} \mathrm{~J}_{6^{\prime}-6}=12.4 \mathrm{~Hz},{ }^{3} \mathrm{~J}_{6^{\prime}-5}=4.8 \mathrm{~Hz}, 1 \mathrm{H}, \mathrm{H}-6\right.$ '), 4.26 (br s, $\left.2 \mathrm{H}, \mathrm{CH}_{2} \mathrm{CONH}\right) 5.06-5.1(\mathrm{~m}$, 
1H, Val $\alpha-\mathrm{CH}) 5.20-5.25(\mathrm{~m}, 1 \mathrm{H}, \mathrm{H}-4) 5.37-5.43(\mathrm{~m}, 1 \mathrm{H}, \mathrm{H}-2) 5.83-5.85(\mathrm{~m}, 1 \mathrm{H}, \mathrm{H}-1) 6.73$ (br s, $1 \mathrm{H}, \mathrm{CONH}) 7.78(\mathrm{~s}$, triazole $\mathrm{CH}) ;{ }^{13} \mathrm{C}$ NMR $\left(100 \mathrm{MHz}, \mathrm{CDCl}_{3}\right): \delta 17.8\left(\mathrm{Val} \mathrm{CH}_{3}\right) 19.5$ (Val $\left.\mathrm{CH}_{3}\right) 20.3$ (OAc) 20.7 (OAc) 20.7 (OAc) 20.9 (OAc) 28.5 (t-Bu) 31.0 (Val i-pr CH) 35.0 $\left(\mathrm{CH}_{2} \mathrm{CONH}\right) 60.0$ (Val $\alpha$-C) 61.70 (C-5) 67.9 (C-6) 70.5 (C-2) 72.8 (C-3) 75.3 (C-4) 86.0 (C-1) $156.1(\mathrm{Boc} \mathrm{C}=\mathrm{O}) 169.0(\mathrm{OAc} \mathrm{C}=\mathrm{O}) 169.5(\mathrm{OAc} \mathrm{C}=\mathrm{O}) 170.2(\mathrm{OAc} \mathrm{C}=\mathrm{O}) 170.7(\mathrm{OAc} \mathrm{C}=\mathrm{O})$ 170.6 (CONH C=O). HRESIMS calculated for $\mathrm{C}_{27} \mathrm{H}_{41} \mathrm{~N}_{5} \mathrm{O}_{12} \mathrm{Na}^{+}:$: 650.264393. Found: 650.26406 .

4-(17 $\alpha$-Estradiol)-1-(2',3', ,', ,6'-tetra- $O$-acetyl- $\beta$-D-glucopyranosyl)]-1,2,3-triazole (14): Pale yellow solid (71\%). Mp: $133-134{ }^{\circ} \mathrm{C} .{ }^{1} \mathrm{H}$ NMR $\left(400 \mathrm{MHz}, \mathrm{CDCl}_{3}\right): \delta 0.45-0.60(\mathrm{~m}, 1 \mathrm{H}, \mathrm{CH})$ $1.02\left(\mathrm{~s}, 3 \mathrm{H}\right.$, estradiol $\left.\mathrm{CH}_{3}\right) 1.20-1.60(\mathrm{~m}, 6 \mathrm{H}$, estradiol) $1.83(\mathrm{~s}, 3 \mathrm{H}, \mathrm{OAc}) 1.85-1.91(\mathrm{~m}, 2 \mathrm{H}$, estradiol $\left.\mathrm{CH}_{2}\right) 1.96-2.12(\mathrm{~m}, 6 \mathrm{H}$, estradiol H) $2.02(\mathrm{~s}, 3 \mathrm{H}, \mathrm{OAc}) 2.05(\mathrm{~s}, 6 \mathrm{H}, 2 \mathrm{xOAc}) 2.46-$ $2.54\left(\mathrm{~m}, 2 \mathrm{H}\right.$, estradiol $\left.\mathrm{CH}_{2}\right) 2.70($ br s, $1 \mathrm{H}, \mathrm{OH}) 2.72-2.81\left(\mathrm{~m}, 2 \mathrm{H}\right.$, estradiol) 3.99 (ddd, ${ }^{3} \mathrm{~J}_{5-4}=$ $\left.10 \mathrm{~Hz}, 3 \mathrm{~J}_{5-6},=4.8,{ }^{3} \mathrm{~J}_{5-6}=2.0 \mathrm{~Hz}, 1 \mathrm{H}, \mathrm{H}-5\right) 4.14\left(\mathrm{dd},{ }^{2} \mathrm{~J}_{6-6},=12.8 \mathrm{~Hz},{ }^{3} \mathrm{~J}_{6-5}=2.0 \mathrm{~Hz}, 1 \mathrm{H}, \mathrm{H}-6\right)$ $4.30\left(\mathrm{dd},{ }^{2} \mathrm{~J}_{6^{\prime} 6}=12.4 \mathrm{~Hz},{ }^{3} \mathrm{~J}_{6^{\prime}-5}=4.8 \mathrm{~Hz}, 1 \mathrm{H}, \mathrm{H}-6^{\prime}\right) 5.22-5.27$ (m, 1H, H-4) $5.37-5.42(\mathrm{~m}, 1 \mathrm{H}$, H-3) $5.44-5.48(\mathrm{~m}, 1 \mathrm{H}, \mathrm{H}-2) 5.86\left(\mathrm{~d},{ }^{3} \mathrm{~J}_{1-2}=8 \mathrm{~Hz}, 1 \mathrm{H}, \mathrm{H}-1\right) 6.52-6.58(\mathrm{~m}, 3 \mathrm{H} 2$ x Ar CH) 7.02 - $7.04(\mathrm{~m}, 1 \mathrm{H}, \mathrm{Ar} \mathrm{CH}) 7.70(\mathrm{~s}, 1 \mathrm{H}$, triazole $\mathrm{CH}) ;{ }^{13} \mathrm{C}$ NMR $\left(100 \mathrm{MHz}, \mathrm{CDCl}_{3}\right): \delta 14.4(\mathrm{C}-18)$ 20.3 (OAc) 20.7 (OAc) 20.8 (OAc) 20.9 (OAc) 23.9 (estradiol) 26.5 (estradiol) 27.5 (estradiol) 29.8 (estradiol) 33.0 (estradiol) 38.20 (estradiol) 39.6 (estradiol) 43.6 (estradiol) 47.6 (estradiol) 48.6 (estradiol) 61.8 (C-6’) 68.0 (C-4') 70.5 (C-2') 72.9 (C-3’) 75.3 (C-5’) 82.8 (estradiol C-17) $85.9(\mathrm{C}-1) 112.9(\mathrm{Ar} \mathrm{CH}) 115.5(\mathrm{Ar} \mathrm{CH}) 120.3($ triazole $\mathrm{CH}) 126.5(\mathrm{Ar} \mathrm{CH}) 132.5(\mathrm{Ar} \mathrm{C}) 138.4$ (Ar C) 153.9 (triazole C) 154.8 (phenol C) 169.1 (OAc) 169.7 (OAc) 170.2 (OAc) 170.9 (OAc). HRESIMS: Calculated for $\mathrm{C}_{34} \mathrm{H}_{43} \mathrm{~N}_{3} \mathrm{O}_{11} \mathrm{Na}^{+}: 692.27898$. Found: 692.279186 . 


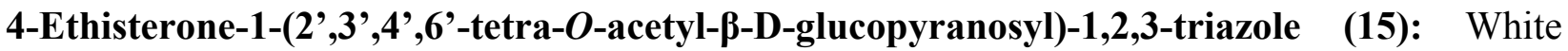
crystalline solid (64\%). Mp: $124-125{ }^{\circ} \mathrm{C} .{ }^{1} \mathrm{H}$ NMR (400 MHz, $\left.\mathrm{CDCl}_{3}\right): \delta 0.32-0.396(\mathrm{~m}, 1 \mathrm{H}$, ethisterone $\mathrm{CH}) 0.65-0.71(\mathrm{~m}, 1 \mathrm{H}$, ethisterone $\mathrm{CH}) 1.04\left(\mathrm{~s}, 3 \mathrm{H}\right.$, ethisterone $\left.\mathrm{CH}_{3}\right) 1.16(\mathrm{~s}, 3 \mathrm{H}$, ethisterone $\left.\mathrm{CH}_{3}\right) 1.33-1.66(\mathrm{~m}, 7 \mathrm{H}$, ethisterone) $2.01(\mathrm{~s}, 3 \mathrm{H}, \mathrm{OAc}) 2.02-2.04(\mathrm{~m}, 4 \mathrm{H}$, ethisterone) $2.05(\mathrm{~s}, 3 \mathrm{H}, \mathrm{OAc}) 2.06(\mathrm{~s}, 6 \mathrm{H}, 2 \times$ OAc) $2.22-2.41(\mathrm{~m}, 5 \mathrm{H}$, ethisterone) $2.44-2.52$ (m, $1 \mathrm{H}$, ethisterone $\mathrm{CH}) 3.99\left(\mathrm{ddd},{ }^{3} \mathrm{~J}_{5-4}=10 \mathrm{~Hz},{ }^{3} \mathrm{~J}_{5-6},=4.8 \mathrm{~Hz},{ }^{3} \mathrm{~J}_{5-6}=2.0 \mathrm{~Hz}, 1 \mathrm{H}, \mathrm{H}-5\right) 4.14$ $\left(\mathrm{dd},{ }^{2} \mathrm{~J}_{6-6},=12.8 \mathrm{~Hz},{ }^{3} \mathrm{~J}_{6-5}=2.0 \mathrm{~Hz}, 1 \mathrm{H}, \mathrm{H}-6\right) 4.30\left(\mathrm{dd},{ }^{2} \mathrm{~J}_{6-6}=12.8 \mathrm{~Hz},{ }^{3} \mathrm{~J}_{6}{ }^{\prime}-5=4.8 \mathrm{~Hz}, 1 \mathrm{H} . \mathrm{H}-6^{\prime}\right)$ $5.20-5.25(\mathrm{~m}, 1 \mathrm{H}, \mathrm{H}-4) 5.36-5.44(\mathrm{~m}, 2 \mathrm{H}, \mathrm{H}-2$ and $\mathrm{H}-3) 5.68(\mathrm{~s}, 1 \mathrm{H}$, ethisterone $\mathrm{C}=\mathrm{CH}) 5.83$ - $5.85(\mathrm{~m}, 1 \mathrm{H}, \mathrm{H}-1) 7.66(\mathrm{~s}, 1 \mathrm{H}$, triazole $\mathrm{CH}) ;{ }^{13} \mathrm{C} \mathrm{NMR}\left(100 \mathrm{MHz}, \mathrm{CDCl}_{3}\right): \delta 14.4($ ethisterone $\mathrm{CH}_{3}$ ) 17.6 (ethisterone $\mathrm{CH}_{3}$ ) 20.3 (OAc) 20.7 (OAc) 20.8 (OAc) 20.9 (OAc) 24.1 (ethisterone) 31.8 (ethisterone) 32.7 (ethisterone) 33.0 (ethisterone) 34.1 (ethisterone) 35.9 (ethisterone) 36.5 (ethisterone) 38.0 (ethisterone) 38.8 (ethisterone) 47.1 (ethisterone) 49.0 (ethisterone) 53.6 (ethisterone) 61.3 (C-6) 68.0 (C-4) 70.5 (C-2 or C-3) 72.8 (C-2 or C-3) 75.4 (C-5) 82.5 (ethisterone C-17) 86.0 (C-1) 119.9 (triazole $\mathrm{CH}$ ) 124.0 (ethisterone $\mathrm{C}=\mathrm{CH}$ ) 154.5 (triazole $\mathrm{C}$ ) 168.9 (OAc) 169.6 (OAc) 170.1 (OAc) 170.7 (ethisterone $C=\mathrm{CH}) 171.4$ (OAc) 199.7 (ethisterone $\mathrm{C}=\mathrm{O}$ ). HRESIMS: calculated for $\mathrm{C}_{35} \mathrm{H}_{47} \mathrm{~N}_{3} \mathrm{O}_{11} \mathrm{Na}^{+}$: 708.31028. Found: 708.310775. Anal. Calculated for $\mathrm{C}_{35} \mathrm{H}_{47} \mathrm{~N}_{3} \mathrm{O}_{11}$ : C, 61.30; H, 6.91; N, 6.13; O, 25.66. Found: C, 61.31; H, 6.99; N, 5.98 .

\section{4-(4-Sulfamoylamidomethyl)-1-(methyl 2',3',4'-tri-O-acetyl- $\beta$-D-glucuronate)-1,2,3-triazole}

(16). Off-white amorphous solid (85\%). Mp (decomp): $221-222{ }^{\circ} \mathrm{C} . R_{f}=0.08$ (8:2 ethyl acetatehexanes). ${ }^{1} \mathrm{H}$ NMR ( $d_{6}$ DMSO, $\left.400 \mathrm{MHz}\right): \delta 1.76$ (s, 3H, OAc) 1.95 (s, 3H, OAc) 1.98 (s, 3H, OAc) $3.59\left(\mathrm{~s}, 3 \mathrm{H}, \mathrm{CO}_{2} \mathrm{CH}_{3}\right) 4.51\left(\mathrm{~d},{ }^{3} \mathrm{~J}_{\mathrm{CH} 2-\mathrm{NH}}=5.2 \mathrm{~Hz}, 2 \mathrm{H}, \mathrm{NHCH}_{2}\right) 4.76\left(\mathrm{~d},{ }^{3} \mathrm{~J}_{5-4}=10 \mathrm{~Hz}, 1 \mathrm{H}\right.$, 
H-5) $5.19-5.24(\mathrm{~m}, 1 \mathrm{H}, \mathrm{H}-4) 5.44-5.59(\mathrm{~m}, 1 \mathrm{H}, \mathrm{H}-3) 5.72-5.76(\mathrm{~m}, 1 \mathrm{H}, \mathrm{H}-2) 6.34\left(\mathrm{~d},{ }^{3} \mathrm{~J}_{1-2}=\right.$ $9.6 \mathrm{~Hz}, 1 \mathrm{H}, \mathrm{H}-1) 7.45\left(\right.$ br s, 2H, $\left.\mathrm{SO}_{2} \mathrm{NH}_{2}\right) 7.86-8.01(\mathrm{~m}, 4 \mathrm{H}, \mathrm{Ar} \mathrm{H}) 8.35(\mathrm{~s}, 1 \mathrm{H}$, triazole $\mathrm{H}) 9.23$ $\left(\mathrm{t},{ }^{3} \mathrm{~J}_{\mathrm{NH}-\mathrm{CH} 2}=5.6 \mathrm{~Hz}, 1 \mathrm{H}, \operatorname{ArCONH}\right) ;{ }^{13} \mathrm{C} \mathrm{NMR}\left(d_{6} \mathrm{DMSO}, 100 \mathrm{MHz}\right): 20.6\left(\mathrm{OAc} \mathrm{CH}_{3}\right) 20.9$ $\left(\mathrm{OAc} \mathrm{CH}_{3}\right) 21.0\left(\mathrm{OAc} \mathrm{CH}_{3}\right) 35.5\left(\mathrm{CH}_{2} \mathrm{NH}\right) 53.3\left(\mathrm{CO}_{2} \mathrm{CH}_{3}\right) 69.0(\mathrm{C}-4) 70.4(\mathrm{C}-2) 72.3(\mathrm{C}-3) 73.5$ (C-5) $84.4(\mathrm{C}-1) 122.9$ (triazole CH) $126.3(\mathrm{Ar} \mathrm{CH}) 128.7(\mathrm{Ar} \mathrm{CH}) 137.6(\mathrm{Ar} \mathrm{C}) 146.4(\mathrm{Ar} \mathrm{C})$ 147.1 (triazole $\mathrm{C}) 165.9(\mathrm{C}=\mathrm{O}) 167.3(\mathrm{C}=\mathrm{O}) 169.1(\mathrm{C}=\mathrm{O}) 170.0(\mathrm{C}=\mathrm{O}) 170.2(\mathrm{C}=\mathrm{O})$. HRESIMS (-ve ion) calculated for $\mathrm{C}_{23} \mathrm{H}_{26} \mathrm{~N}_{5} \mathrm{O}_{12} \mathrm{~S}^{-}$: 596.130416. Found: 596.132499. Anal. Calculated for $\mathrm{C}_{23} \mathrm{H}_{27} \mathrm{~N}_{5} \mathrm{O}_{12} \mathrm{~S}: \mathrm{C}, 46.23 ; \mathrm{H}, 4.55 ; \mathrm{N}, 11.72 ; \mathrm{O}, 32.13 ; \mathrm{S}, 5.37$. Found: $\mathrm{C}, 46.10 ; \mathrm{H}, 4.66 ; \mathrm{N}$, 11.51 .

4-Methylenebenzotriazole-1-(2',3',4'-tri-O-acetyl- $\alpha$-D-arabinosyl)-1,2,3-triazole (17). White solid (94\%). Mp: $215-216{ }^{\circ} \mathrm{C} .{ }^{1} \mathrm{H}$ NMR (400 MHz, $\mathrm{CDCl}_{3}$ ): $\delta 1.80$ (s, 3H, OAc) 1.98 (s, 3H, OAc) $2.17(\mathrm{~s}, 3 \mathrm{H}, \mathrm{OAc}) 3.87\left(\mathrm{dd},{ }^{2} \mathrm{~J}_{5-5},=13.6,{ }^{3} \mathrm{~J}_{5-4}=1.2 \mathrm{~Hz}, 1 \mathrm{H}, \mathrm{H}-5\right) 4.11\left(\mathrm{dd},{ }^{2} \mathrm{~J}_{5^{\prime}-5}=13.6 \mathrm{~Hz}\right.$, $\left.{ }^{3} \mathrm{~J}_{5^{\prime}-4}=2 \mathrm{~Hz}, 1 \mathrm{H}, \mathrm{H}-5^{\prime}\right) 5.19\left(\mathrm{dd},{ }^{3} \mathrm{~J}_{3-2}=10 \mathrm{~Hz},{ }^{3} \mathrm{~J}_{3-4}=3.2 \mathrm{~Hz}, 1 \mathrm{H}, \mathrm{H}-3\right) 3.58\left(\mathrm{ddd},{ }^{3} \mathrm{~J}_{4-3}=3.6 \mathrm{~Hz}\right.$, $\left.{ }^{3} \mathrm{~J}_{4-5}=2 \mathrm{~Hz},{ }^{3} \mathrm{~J}_{4-5}=1.2 \mathrm{~Hz}, 1 \mathrm{H}, \mathrm{H}-4\right) 5.43-5.48(\mathrm{~m}, 1 \mathrm{H}, \mathrm{H}-2) 5.66\left(\mathrm{~d},{ }^{3} \mathrm{~J}_{1-2}=11.2 \mathrm{~Hz}, 1 \mathrm{H}, \mathrm{H}-1\right)$ $5.92-6.03\left(\mathrm{~m}, 2 \mathrm{H}, \mathrm{Bt}_{-} \mathrm{CH}_{2}\right) 7.33-7.37(\mathrm{~m}, 1 \mathrm{H}, \mathrm{Ar} \mathrm{H}) 7.43-7.47(\mathrm{~m}, 1 \mathrm{H}, \mathrm{Ar} \mathrm{H}) 7.64-7.66(\mathrm{~m}$, 1H Ar H) $7.81(\mathrm{~s}$, triazole $\mathrm{CH}) 8.03-8.05(\mathrm{~m}, 1 \mathrm{H}, \mathrm{Ar} \mathrm{H}) ;{ }^{13} \mathrm{C} \mathrm{NMR}\left(100 \mathrm{MHz}, \mathrm{CDCl}_{3}\right) \delta 20.3$ (OAc) $20.7(\mathrm{OAc}) 21.1(\mathrm{OAc}) 43.9\left(\mathrm{Bt}-\mathrm{CH}_{2}\right) 67.5(\mathrm{C}-5) 67.8(\mathrm{C}-4) 68.4(\mathrm{C}-2) 70.5(\mathrm{C}-3) 87.0$ (C-1) $110.2(\mathrm{Ar} \mathrm{CH}) 120.1(\mathrm{Ar} \mathrm{CH}) 121.9($ triazole $\mathrm{CH}) 124.3(\mathrm{Ar} \mathrm{CH}) 127.9(\mathrm{Ar} \mathrm{CH}) 169.1$ (OAc) $170.0(\mathrm{OAc})$ 170.3 (OAc); HRESIMS calculated for $\mathrm{C}_{20} \mathrm{H}_{22} \mathrm{~N}_{6} \mathrm{O}_{7} \mathrm{Na}^{+}$: 481.144218 . Found: 481.14411. Anal. Calculated for $\mathrm{C}_{20} \mathrm{H}_{22} \mathrm{~N}_{6} \mathrm{O}_{7}$ : C, 52.40; H, 4.84; N, 18.33; O, 24.43. Found: C, 52.39; H, 4.94; N, 18.05; O, 24.62. 


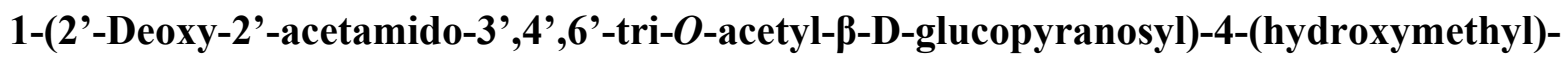

1,2,3-triazole (18). ${ }^{16 \mathrm{f}}$ Off-white foam (62\%). ${ }^{1} \mathrm{H}$ NMR (400 MHz, $d_{6}$ DMSO): $\delta 1.57$ (s, 3H, NHAc) $1.92(\mathrm{~s}, 3 \mathrm{H}, \mathrm{OAc}) 1.97(\mathrm{~s}, 3 \mathrm{H}, \mathrm{OAc}) 1.98(\mathrm{~s}, 3 \mathrm{H}, \mathrm{OAc}) 3.99-4.03\left(\mathrm{dd},{ }^{2} \mathrm{~J}_{6-6},=12.4 \mathrm{~Hz}\right.$,

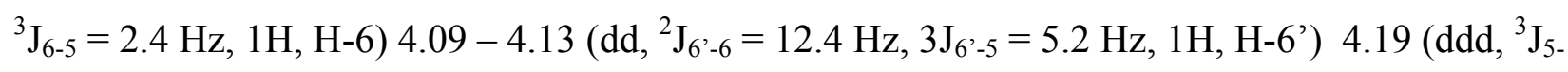
$\left.{ }_{4}=10 \mathrm{~Hz},{ }^{3} \mathrm{~J}_{5-6},=4.8 \mathrm{~Hz},{ }^{3} \mathrm{~J}_{5-6}=2.4 \mathrm{~Hz}, 1 \mathrm{H}, \mathrm{H}-5\right) 4.47\left(\mathrm{~d},{ }^{3} \mathrm{~J}_{\mathrm{CH} 2-\mathrm{OH}}=4.4 \mathrm{~Hz}, 2 \mathrm{H}, \mathrm{CH}_{2} \mathrm{OH}\right) 5.04-$ $5.09(\mathrm{~m}, 1 \mathrm{H}, \mathrm{H}-3) 5.20-5.21\left(\mathrm{~m}, 1 \mathrm{H}, \mathrm{CH}_{2} \mathrm{OH}\right) 5.29-5.34(\mathrm{~m}, 1 \mathrm{H}, \mathrm{H}-4) 6.06\left(\mathrm{~d},{ }^{3} \mathrm{~J}_{1-2}=10 \mathrm{~Hz}\right.$, $1 \mathrm{H}, \mathrm{H}-1) 8.04\left(\mathrm{~d},{ }^{3} \mathrm{~J}_{\mathrm{NH}-2}=9.6 \mathrm{~Hz}, 1 \mathrm{H}, \mathrm{NHAc} \mathrm{NH}\right) 8.09(\mathrm{~s}, 1 \mathrm{H}$, triazole $\mathrm{CH}) ;{ }^{13} \mathrm{C}$ NMR $(100$ MHz, d $d_{6}$ DMSO): $\delta 25.7$ (OAc) 25.9 (OAc) 26.0 (OAc) 27.8 (NHAc) $57.4(\mathrm{C}-2) 60.0\left(\mathrm{CH}_{2} \mathrm{OH}\right)$ 67.3 (C-4) 73.4 (C-5) 77.9 (C-3) 78.7 (C-6) 90.0 (C-1) 126.8 (triazole CH) 153.6 (triazole C) $174.8(\mathrm{C}=\mathrm{O}) 174.9(\mathrm{C}=\mathrm{O}) 175.0(\mathrm{C}=\mathrm{O}) 175.5(\mathrm{C}=\mathrm{O})$. HRESIMS calculated for $\mathrm{C}_{17} \mathrm{H}_{24} \mathrm{~N}_{4} \mathrm{O}_{9} \mathrm{Na}^{+}$: 541.143549. Found: 451.142863.

4-Hydroxymethyl-1-(2',3',4',6'-tetra-O-benzyl-a-D-glucopyranosyl)-1,2,3-triazole (19). To a vigorously stirring solution of 2,3,4,6-tetra-O-benzyl- $\alpha$-D-glucopyranosyl azide (100 mg, 0.18 mmol) in hot tert-butyl alcohol $\left(500 \mu \mathrm{L}\right.$ at ca. $\left.60{ }^{\circ} \mathrm{C}\right)$, was added propargyl alcohol $(45 \mu \mathrm{L}, 0.74$ mmol, 4.2 equiv) and a solution of $\mathrm{CuSO}_{4} 5 \mathrm{H}_{2} \mathrm{O}(18 \mathrm{mg}, 0.08 \mathrm{mmol}, 0.4$ equiv) and sodium ascorbate (30 mg, $0.15 \mathrm{mmol}, 0.8$ equiv) in distilled $\mathrm{H}_{2} \mathrm{O}(500 \mu \mathrm{L})$. The deep yellow suspension was stirred at $60{ }^{\circ} \mathrm{C}$ for approx. $2 \mathrm{~d}$, at which time TLC indicated reaction completion (1:1 ethyl acetate:hexanes). The aqueous phase was then extracted with $\mathrm{CH}_{2} \mathrm{Cl}_{2}(2 \mathrm{x} 10 \mathrm{~mL})$, and the combined organic extracts were dried $\left(\mathrm{MgSO}_{4}\right)$, filtered and evaporated to afford a pale yellow syrup, which crystallized on standing to afford a white amorphous solid. (79 mg, 73 \%), Mp: 82 $83{ }^{\circ} \mathrm{C} . R_{f}=0.19 .{ }^{1} \mathrm{H}$ NMR $\left(400 \mathrm{MHz}, \mathrm{CDCl}_{3}\right): 3.51\left(\mathrm{dd},{ }^{2} \mathrm{~J}_{6-6},=10.8 \mathrm{~Hz},{ }^{3} \mathrm{~J}_{6-6}=2.0 \mathrm{~Hz}, 1 \mathrm{H}, \mathrm{H}-6\right)$ $3.67\left(\mathrm{dd},{ }^{2} \mathrm{~J}_{6^{\prime}-6}=10.8 \mathrm{~Hz},{ }^{3} \mathrm{~J}_{6^{\prime}-5}=2.8 \mathrm{~Hz}, 1 \mathrm{H}, \mathrm{H}-6{ }^{\prime}\right) 3.81\left(\mathrm{dd},{ }^{3} \mathrm{~J}=10 \mathrm{~Hz}, 3 \mathrm{~J}=8.4 \mathrm{~Hz}, 1 \mathrm{H}, \mathrm{H}-4\right)$ 
$3.89-3.93(\mathrm{~m}, 1 \mathrm{H}, \mathrm{H}-5) 4.02\left(\mathrm{dd},{ }^{3} \mathrm{~J}_{2-3}=9.6 \mathrm{~Hz},{ }^{3} \mathrm{~J}_{2-1}=6 \mathrm{~Hz}, 1 \mathrm{H}, \mathrm{H}-2\right) 4.39-4.53(\mathrm{~m}, 2 \mathrm{H}, \mathrm{OBn}$ $\left.\mathrm{CH}_{2}\right) 4.49-4.73\left(\mathrm{~m}, 2 \mathrm{H}, \mathrm{OBn} \mathrm{CH}_{2}\right) 4.52-4.85\left(\mathrm{~m}, 2 \mathrm{H}, \mathrm{OBn} \mathrm{CH}_{2}\right) 4.60-4.64(\mathrm{~m}, 1 \mathrm{H}, \mathrm{H}-3)$ $4.80\left(\mathrm{~s}, 2 \mathrm{H}, \mathrm{OCH}_{2}\right) 4.84-4.94(\mathrm{~m}, 2 \mathrm{H}, \mathrm{OBn} \mathrm{CH} 2) 5.80\left(\mathrm{~d},{ }^{3} \mathrm{~J}_{1-2}=5.6 \mathrm{~Hz}, 1 \mathrm{H}, \mathrm{H}-1\right) 7.13-7.16$ (m, 4H, OBn CH) $7.25-7.33(\mathrm{~m}, 16 \mathrm{H}, \mathrm{OBn} \mathrm{CH}) 7.51$ (s, triazole $\mathrm{CH}) ;{ }^{13} \mathrm{C}$ NMR $(100 \mathrm{MHz}$, $\left.\mathrm{CDCl}_{3}\right): 56.8\left(\mathrm{OCH}_{2}\right) 68.3(\mathrm{C}-6) 73.7(\mathrm{C}-5) 73.9\left(\mathrm{OBn} \mathrm{CH}_{2}\right) 74.4\left(\mathrm{OBn} \mathrm{CH}_{2}\right) 75.0\left(\mathrm{OBn} \mathrm{CH}_{2}\right)$ $75.8\left(\mathrm{OBn} \mathrm{CH}_{2}\right) 77.4(\mathrm{C}-4) 78.8(\mathrm{C}-2) 82.0(\mathrm{C}-3) 84.3(\mathrm{C}-1) 124.0$ (triazole $\left.\mathrm{CH}\right) 127.9(\mathrm{OBn}$ CH) $127.93(\mathrm{OBn} \mathrm{CH}) 127.97(\mathrm{OBn} \mathrm{CH}) 128.1(\mathrm{OBn} \mathrm{CH}) 128.2(\mathrm{OBn} \mathrm{CH}) 128.4(\mathrm{OBn} \mathrm{CH})$ $128.5(\mathrm{OBn} \mathrm{CH}) 128.59(\mathrm{OBn} \mathrm{CH}) 128.62(\mathrm{OBn} \mathrm{CH}) 128.7(\mathrm{OBn} \mathrm{CH}) 128.9(\mathrm{OBn} \mathrm{CH}) 137.7$ (OBn C) 137.8 (OBn C) 138.3 (OBn C) 138.7 (OBn C). HRESIMS calculated for $\mathrm{C}_{37} \mathrm{H}_{39} \mathrm{~N}_{3} \mathrm{O}_{6} \mathrm{Na}^{+}: 644.273116$. Found: 644.272986.

\section{Methyl 2,3,4-tri-O-acetyl-6-deoxy-6-(4-\{[(2,3,4,6-tetra-O-acetyl- $\beta$-D-glucopyranosyl)oxy]} methyl\}-1H-1,2,3-triazol-1-yl)- $\alpha$-D-glucopyranoside. (20): White foam (78\%). ${ }^{1} \mathrm{H}$ NMR (400 $\left.\mathrm{MHz}, \mathrm{CDCl}_{3}\right): \delta 1.96(\mathrm{~s}, 3 \mathrm{H}, \mathrm{OAc}) 1.99(\mathrm{~s}, 6 \mathrm{H}, 2$ x OAc) $2.00(\mathrm{~s}, 3 \mathrm{H}, \mathrm{OAc}) 2.04(\mathrm{~s}, 3 \mathrm{H}, \mathrm{OAc})$ $2.08(\mathrm{~s}, 6 \mathrm{H}, 2 \times \mathrm{OAc}) 3.14\left(\mathrm{~s}, 3 \mathrm{H}, \mathrm{OCH}_{3}\right) 3.72\left(\mathrm{ddd},{ }^{3} \mathrm{~J}_{5-4}=10 \mathrm{~Hz},{ }^{3} \mathrm{~J}_{5-6}=5.2 \mathrm{~Hz}, 3 \mathrm{~J}_{5-6}=2.8 \mathrm{~Hz}\right.$, $1 \mathrm{H}, \beta \mathrm{Glc} \mathrm{H}-5) 4.14\left(\mathrm{dd},{ }^{2} \mathrm{~J}_{6-6},=12.4 \mathrm{~Hz},{ }^{3} \mathrm{~J}_{6-5}=2.4 \mathrm{~Hz}, 1 \mathrm{H} \beta \mathrm{Glc} \mathrm{H}-6\right) 4.17-4.20$ (m, 1H, $\alpha \mathrm{Glc}$ H-5) $4.26\left(\mathrm{dd}^{2}{ }^{2} \mathrm{~J}_{6^{\prime}-6}=12.4 \mathrm{~Hz},{ }^{3} \mathrm{~J}_{6^{\prime}-5}=4.62,1 \mathrm{H}, \beta \mathrm{Glc} \mathrm{H}-6^{\prime}\right) 4.39\left(\mathrm{dd},{ }^{2} \mathrm{~J}_{6-6^{\prime}}=14.4 \mathrm{~Hz},{ }^{3} \mathrm{~J}_{6-5}=8.4\right.$ $\mathrm{Hz}, 1 \mathrm{H}, \alpha \mathrm{Glc} \mathrm{H}-6) 4.56\left(\mathrm{dd},{ }^{2} \mathrm{~J}_{6^{\prime}-6}=14.6 \mathrm{~Hz},{ }^{3} \mathrm{~J}_{6^{\prime}-5}=2.4 \mathrm{~Hz}, 1 \mathrm{H}, \alpha \mathrm{Glc} \mathrm{H}-6^{\prime}\right) 4.52\left(\mathrm{~d},{ }^{3} \mathrm{~J}_{1-2}=8.4\right.$ $\mathrm{Hz}, 1 \mathrm{H}, \beta \mathrm{Glc} \mathrm{H}-1) 4.73-4.82$ (m, 3H, $\left.\alpha \mathrm{Glc} \mathrm{H}-2, \alpha \mathrm{Glc} \mathrm{H}-4, \mathrm{CH}_{2} \mathrm{O}\right) 4.89\left(\mathrm{~d},{ }^{3} \mathrm{~J}_{1-2}=3.6 \mathrm{~Hz}, 1 \mathrm{H}\right.$, $\alpha$ Glc H-1) 4.99 (dd, $\left.{ }^{3} \mathrm{~J}=9.2 \mathrm{~Hz},{ }^{3} \mathrm{~J}_{2-1}=8 \mathrm{~Hz}, 1 \mathrm{H}, \beta \mathrm{Glc} \mathrm{H}-2\right) 5.05-5.09$ (m, 1H, $\left.\beta \mathrm{Glc} \mathrm{H}-4\right) 5.14$ $-5.19(\mathrm{~m}, 1 \mathrm{H}, \alpha \mathrm{Glc} \mathrm{H}-3) 7.16(\mathrm{~s}, 1 \mathrm{H}$, triazole $\mathrm{H}) ;{ }^{13} \mathrm{C}$ NMR $\left(100 \mathrm{MHz}, \mathrm{CDCl}_{3}\right): \delta 50.9(\beta \mathrm{Glc} \mathrm{C}-$ 5) $55.8\left(\mathrm{OCH}_{3}\right) 62.0(\beta \mathrm{Glc} \mathrm{C}-6) 62.9\left(\mathrm{CH}_{2} \mathrm{O}\right) 67.8(\alpha \mathrm{Glc} \mathrm{C}-5) 68.5(\beta \mathrm{Glc} \mathrm{C}-4) 69.9(\alpha \mathrm{Glc} \mathrm{C}-3)$ 70.0 ( $\alpha$ Glc C-2) 70.9 ( $\alpha$ Glc C-4) 71.3 ( $\beta$ Glc C-2) 72.1 ( $\beta$ Glc H-5) 72.9 ( $\alpha$ Glc C-3) 96.9 ( $\alpha$ Glc C- 
1) 99.7 (BGlc C-1) 124.6 (triazole $\mathrm{CH}) 144.4$ (triazole C) $169.5(\mathrm{OAc}) 169.6(\mathrm{OAc}) 170.0(\mathrm{OAc})$ $170.2(\mathrm{OAc}) 170.4(\mathrm{OAc}) 170.5(\mathrm{OAc}) 170.9$ (OAc). HRESIMS calculated for $\mathrm{C}_{30} \mathrm{H}_{41} \mathrm{~N}_{3} \mathrm{O}_{18} \mathrm{Na}^{+}$: 754.227733. Found 754.226711. Anal. Calculated for $\mathrm{C}_{30} \mathrm{H}_{41} \mathrm{~N}_{3} \mathrm{O}_{18}$ : C, 49.25; H, 5.65; N, 5.74; O, 39.36; Found: C, 48.92; H, 5.71; N, 5.49.

\section{Acknowledgements}

The authors gratefully acknowledge financial support from the Australian Research Council as well as the Eskitis Institute for Cell and Molecular Therapies and the School of Science, GU.

\section{Supplementary data available}

${ }^{1} \mathrm{H}$ NMR (400 MHz) spectra for compounds $\mathbf{2 - 2 0}$ are provided in the Supplementary data.

\section{References}

1. For a series of excellent reviews, see: Sinaÿ, P., Hart, G.W., Ernst, B. Carbohydrates in Chemistry and Biology; Wiley VCH: Weinheim, Germany, 2002.

2. (a) Spiro, R. G. Glycobiology 2002, 12, 43R. (b) Daines, A. M.; Macmillan, D. Curr. Med. Chem. 2003, 10, 2733-2773.

3. (a) Dube, D. H.; Bertozzi, C. R. Nature Rev. Drug Disc. 2005, 4, 481. (b) Prescher, J. A.; Bertozzi, C. R. Nature Chem. Biol. 2005, 1, 13-21.

4. (a) Fernandes B.; Sagman U.; Auger M.; Demetrio M.; Dennis J. W. Cancer Res. 1991, 51, 718-723. (b) Ohyama, C.; Tsuboi, S.; Fukuda, M. EMBO J. 1999, 18, 1516-1525.

5. (a) Feizi, T.; Larkin, M. Glycobiology 1990, 1, 17-23. (b) Pichlmaier, H.; Uhlenbruck, H.; Pulverer, G.; Ko, G.; Beuth, J. Glycoconjugate. J. 1995, 12, 1-6. (c) Schofield, L.; Hewitt, M. C.; Evans, K.; Siomos, M.-A.; Seeberger, P. H. Nature 2002, 418, 785-789.

6. Wharton, S. A., Weis, W., Skehel, D. C., Wiley, D. C., The Influenza Virus; Plenum: New York, 1989.

7. (a) Varki, A. Essentials of Glycobiology $2^{\text {nd }}$ ed.; Cold Spring Harbor Laboratory Press: New York, 1999. (b) Ellgaard, L.; Molinari, M.; Helenius, A. Science 1999, 286, 1882-1888. (c) Roth, J. Chem. Rev. 2002, 102, 285-303. 
8. (a) Herzner, H.; Reipen, T.; Schultz, M.; Kunz, H. Chem. Rev. 2000, 100, 4495-4537. (b) Kiefel, M. J.; von Itzstein, M. Chem. Rev. 2002, 102, 471-490. (c) Davis, B. G. Chem. Rev. 2002, 102, 579-601.

9. Schriver, Z.; Raguram, S.; Sasisekharan, R. Nature Rev. Drug Disc. 2004, 3, 863-873.

10. (a) Lillelund, V. H., Jensen, H. H., Liang, X., Bols, M. Chem. Rev. 2002, 102, 515-553. (b) Houston, T. A.; Blanchfield, J. T. Mini-Rev. Med. Chem. 2003, 3, 669-678. See also 16(c, d).

11. Fan, W.-Q., Katritzky, A. R., In Comprehensive Heterocyclic Chemistry II Katritzky, A. R., Rees, C. W., Scriven, E. F. V., Eds.; Elsevier Science: Oxford, 1996; Vol. 4, pp 1-126. For recent applications of 1,3-DCR to carbohydrates see: (a) Mishra, R. M.; Tewari, N.; Verma, S. S.; Tripathi, R. P.; Kumar, M.; Shukla, P. K. J. Carbohydr. Chem. 2004, 23, 353-374. (b) Perez-Balderas, F., Hernandez-Mateo, F., Santoyo-Gonzalez, F. Tetrahedron, 2005, 61, 93389348.

12. (a) Rostovsev, V. V.; Green, L. G.; Fokin, V. V.; Sharpless, K. B., Angew. Chem. Int. Ed. 2002, 41, 2596-2599. (b) For original definition of "click" chemistry, see: Kolb, H. C.; Finn, M. G.; Sharpless, K. B., Angew. Chem. Int. Ed. 2001, 40, 2004-2021.

13. Tornøe, C.W.; Christensen, C.; Meldal, M. J. Org. Chem. 2002, 67, 3057-3064.

14. (a) Wang, Q.; Chan, T. R.; Hilgraf, R.; Fokin, V. V.; Sharpless, K. B.; Finn, M. G. J. Am. Chem. Soc. 2003, 125, 3192-3193. (b) Horne, W. S.; Stout, D. C.; Ghadiri, M. R.; J. Am. Chem. Soc. 2003, 125, 9372-9376. (c) Lee, L. V., Mitchell, M. L.; Huang, S.-J.; Fokin, V. V.; Sharpless, K. B.; Wong, C.-H. J. Am. Chem. Soc. 2003, 125, 9588-9589. (d) Schultz, P. G.; Anderson, C.; Chin, J. W.; Mukherji, M.; Cropp, A.; Deiters, A. J. Am. Chem. Soc. 2003, 125, 111782-111783. (e) Mantesch, R.; Krasinski, A.; Radic, Z.; Raushel, J.; Taylor, P.; Sharpless, K. B.; Kolb, H. C.; J. Am. Chem. Soc. 2004, 126, 12809-12818. See also: Lewis, W. G.; Green, L. G.; Grynszpan, F.; Radic, Z.; Carlier, P. R.; Taylor, P.; Finn, M. G.; Sharpless, K. B. Angew. Chem. Int. Ed. 2002, 41, 1053-1057.

15. (a) Wu, P.; Feldman, A. K.; Nugent, A. K.; Hawker, C. J.; Scheel, A.; Voit, B.; Pyun, J.; Fréchet, J. M. J.; Sharpless, K. B.; Fokin, V. V. Angew. Chem. Int. Ed. 2004, 43, 3928-3932. (b) Malkoch, M.; Thibault, R.J.; Drockenmuller, E.; Messerschmidt, M.; Voit, B.; Russell, T.P.; Hawker, C.J. J. Am. Chem. Soc. 2005, 127, 14942-14949.

16. (a) Kuijpers, B. H. M.; Groothuys, S.; Keereweer, A. R.; Quaedflieg, P. J. L. M.; Blaauw, R. H.; van Delft, F. L.; Rutjes, F. P. J. T., Org. Lett. 2004, 6, 3123-3126. (b) Chittaboina, S.; 
Xie, F.; Wang, Q. Tetrahedron Lett. 2005, 46, 2331-2336. (c) Rossi, L. L.; Basu, A. Bioorg. Med. Chem. Lett. 2005, 15, 3596-3599. (d) Périon, R.; Ferrières, V.; García-Moreno, M. I.; Ortiz Mellet, C.; Duval, R.; Garcia Fernández, J. M.; Plusquellec, D. Tetrahedron 2005, 61, 9118-9128. (e) Hotha, S.; Kashyap, S. J. Org. Chem. 2006, 71, 364-367. For examples of thermal Huisgen reactions on glycosyl azides see: (f) de la Heras, F. G.; Alonso, R.; Alonso, G. J. Med. Chem. 1979, 22, 496-501. (g) Bröder, W.; Kunz, H. Synlett 1990, 251-252. (h) Marco-Contelles, J.; Jiménez, C. A. Tetrahedron 1999, 55, 10511-10526. (i) Al-Masoudi, N. A.; Al-Soud, Y. A. Tetrahedron Lett. 2002, 43, 4021-4022.

17. Kunz, H.; Waldmann, H. Angew. Chem. Int. Ed. 1985, 24, 883-885.

18. (a) Nicolaou, K. C.; Ladduwahetty, T.; Randall, J. L.; Chucholowski, A.; J. Am. Chem. Soc. 1986, 108, 2466-2468. (b) Broder, W.; Kunz, H. Bioorg. Med. Chem. 1997, 5, 1-19.

19. Alper, P. B.; Hung, S.-C.; Wong, C.-H. Tetrahedron Lett. 1996, 37, 6029-6032.

20. Mereyala, H. B.; Gurrala, S. R. Carbohydr. Res. 1998, 307, 351-354.

21. Akula, R. A.; Artis, N. D.; Temmkoff, D. P.; Norris, P. Heterocycles 2004, 63 (12), 27192725.

22. Wilkinson, B. L.; Bornaghi, L. F.; Houston, T. A.; Poulsen, S.-A. manuscript in preparation.

23. Wilkinson, B. L.; Bornaghi, L. F.; Houston, T. A.; Poulsen, S.-A.; Healy, P. C. Acta Cryst. 2005, E61, o738-0740.

24. Bodine, K. D.; Gin, D. Y.; Gin, M. S. Org. Lett. 2005, 7, 4479-4482. Note the $\alpha$-azides in the mannose derivatives used here also react sluggishly as we observed in the synthesis of 19.

25. (a) Pérez-Balderas, F.; Ortega-Muñoz, M.; Morales-Sanfrutos, J.; Hernández-Mateo, F.; Calvo-Flores, F. G.; Calvo-Asín, J. A.; Isac-García, J.; Santoyo-González, F. Org. Lett. 2003, 5, 1951-1954. (b) Rijkers, D. T. S.; van Esse, G. W.; Merkx, R.; Brouwer, A. J.; Jacobs, H. J. F.; Pieters, R. J.; Liskamp, R. M. J. Chem. Commun. 2005, 4581-4583.

26. Ladmiral, V.; Mantovani, G.; Clarkson, G. J.; Cauet, S.; Irwin, J. L.; Haddleton, D. M. J. Am. Chem. Soc. 2006, 128, 4823-4830.

27. (a) Koreeda, M.; Houston, T. A.; Shull, B. K.; Klemke, E.; Tuinman, R. J. Synlett 1995, 9092. (b) Blanchfield, J.; Toth, I. Curr. Med. Chem. 2004, 11, 2375-2382.

28. Zhang, L.; Chen, X. G.; Xue, P.; Sun, H. H. Y.; Williams, I. D.; Sharpless, K. B.; Fokin, V. V.; Jia, G. C. J. Am. Chem. Soc. 2005, 127, 15998-15999. 


\section{Captions}

Scheme 1. $\mathrm{Cu}(\mathrm{I})$ catalyzed 1,3-dipolar cycloaddition reaction of azides and terminal alkynes.

Scheme 2. Protecting group and glycosylation chemistry on model glucosyl triazole 2. ${ }^{\text {a }}$ 
Table 1. Study of formation of glycosyl triazole $2^{a}$

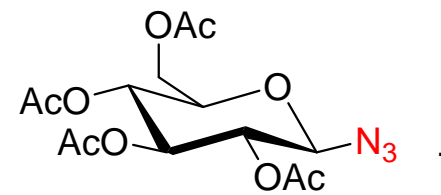

1

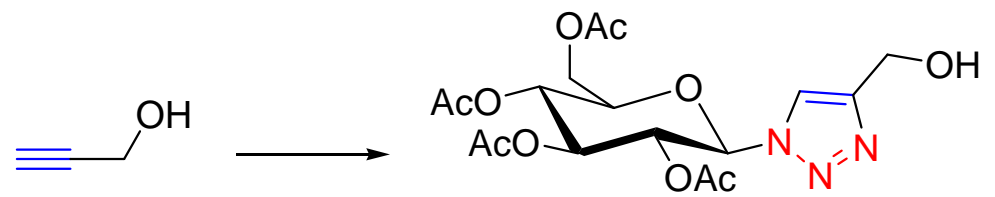

2

\begin{tabular}{ccccc}
\hline entry & co-solvent & temp $\left({ }^{\mathbf{0}} \mathbf{C}\right)$ & time (h) & yield $\boldsymbol{b}(\%)^{(\%)}$ \\
\hline 1 & methanol & 40 & 2 & 79 \\
2 & ethanol & 40 & 2 & 88 \\
3 & isopropanol & 40 & 2 & 91 \\
4 & tert-butanol & 40 & 2 & 92 \\
5 & propargyl & & & 85 \\
6 & tert-butanol & 25 & 8 & 90 \\
\hline
\end{tabular}

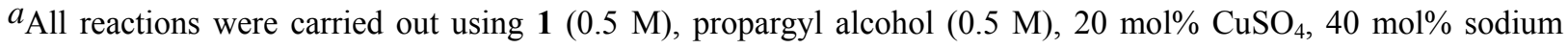
ascorbate (relative to substrate) in 1:1 water/alcohol (with the exception of entry 5). ${ }^{b_{\text {Y }}}$ ield calculated following liquid-liquid extraction into $\mathrm{CH}_{2} \mathrm{Cl}_{2}$. 
Table 2. Examples of glycosyl triazoles

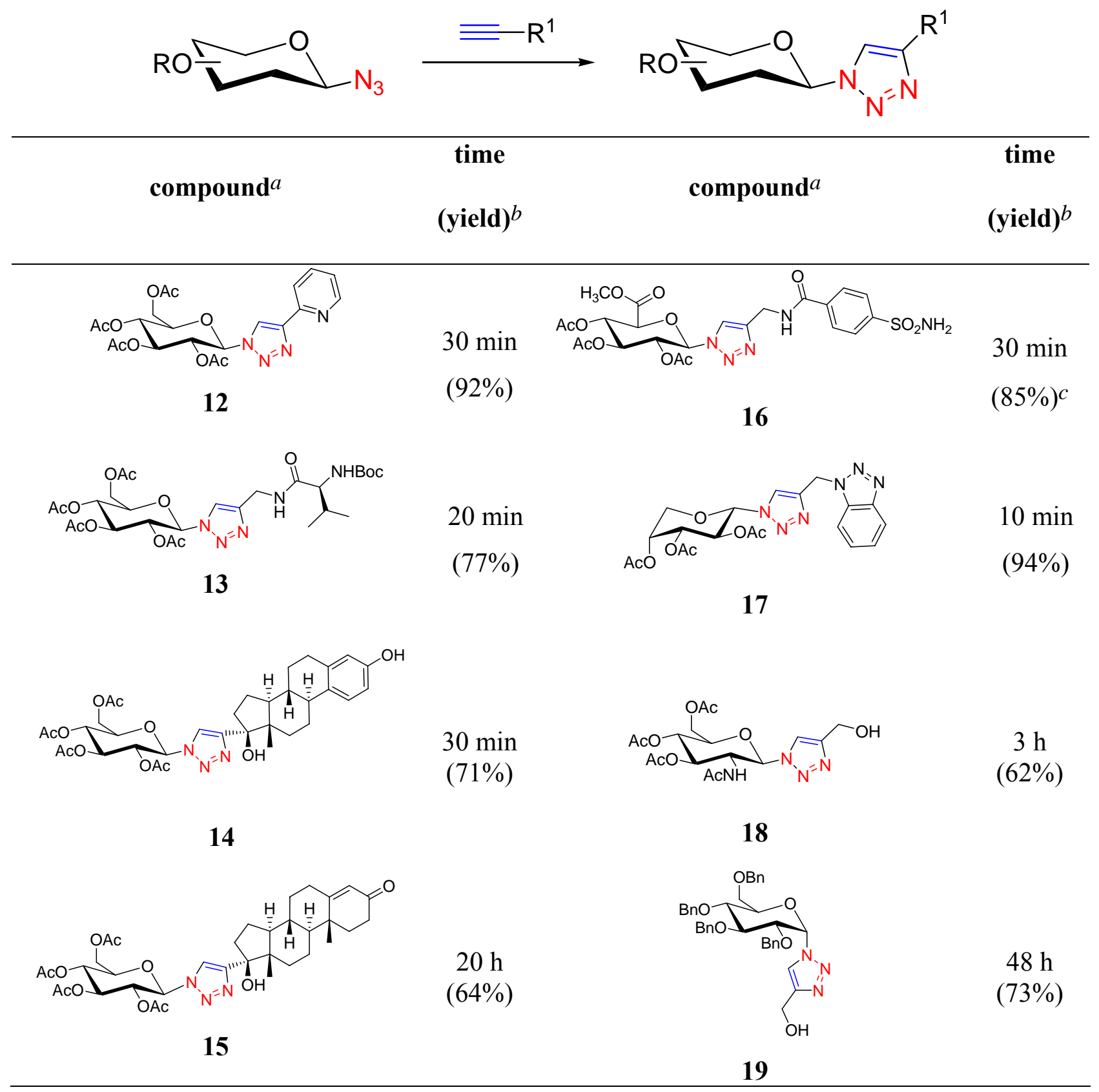

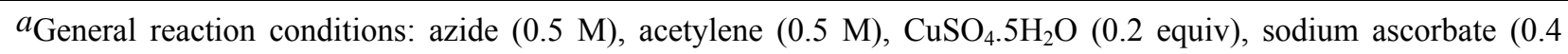
equiv), 1:1 t-BuOH$/ \mathrm{H}_{2} \mathrm{O}, 40{ }^{\circ} \mathrm{C}$, with the exception of 19 , which used acetylene (4.2 equiv), $\mathrm{CuSO}_{4} .5 \mathrm{H}_{2} \mathrm{O}(0.4$ equiv), sodium ascorbate ( 0.8 equiv) at $60{ }^{\circ} \mathrm{C} .{ }^{b}$ Standard purification as described in the Experimental. ${ }^{c}$ Molar yield calculated after precipitation and filtration. 
Scheme 1.

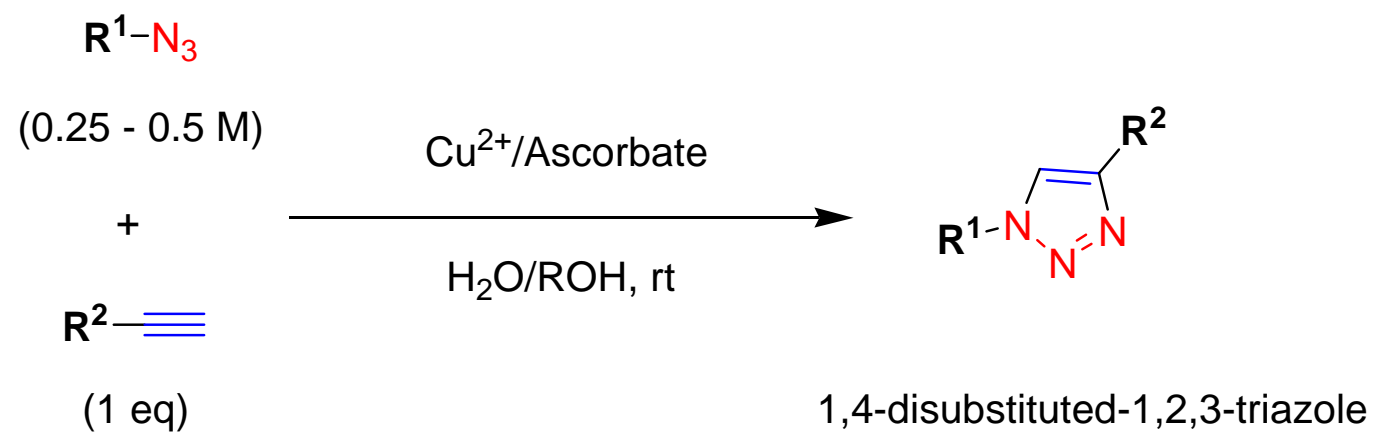




\section{Scheme 2.}

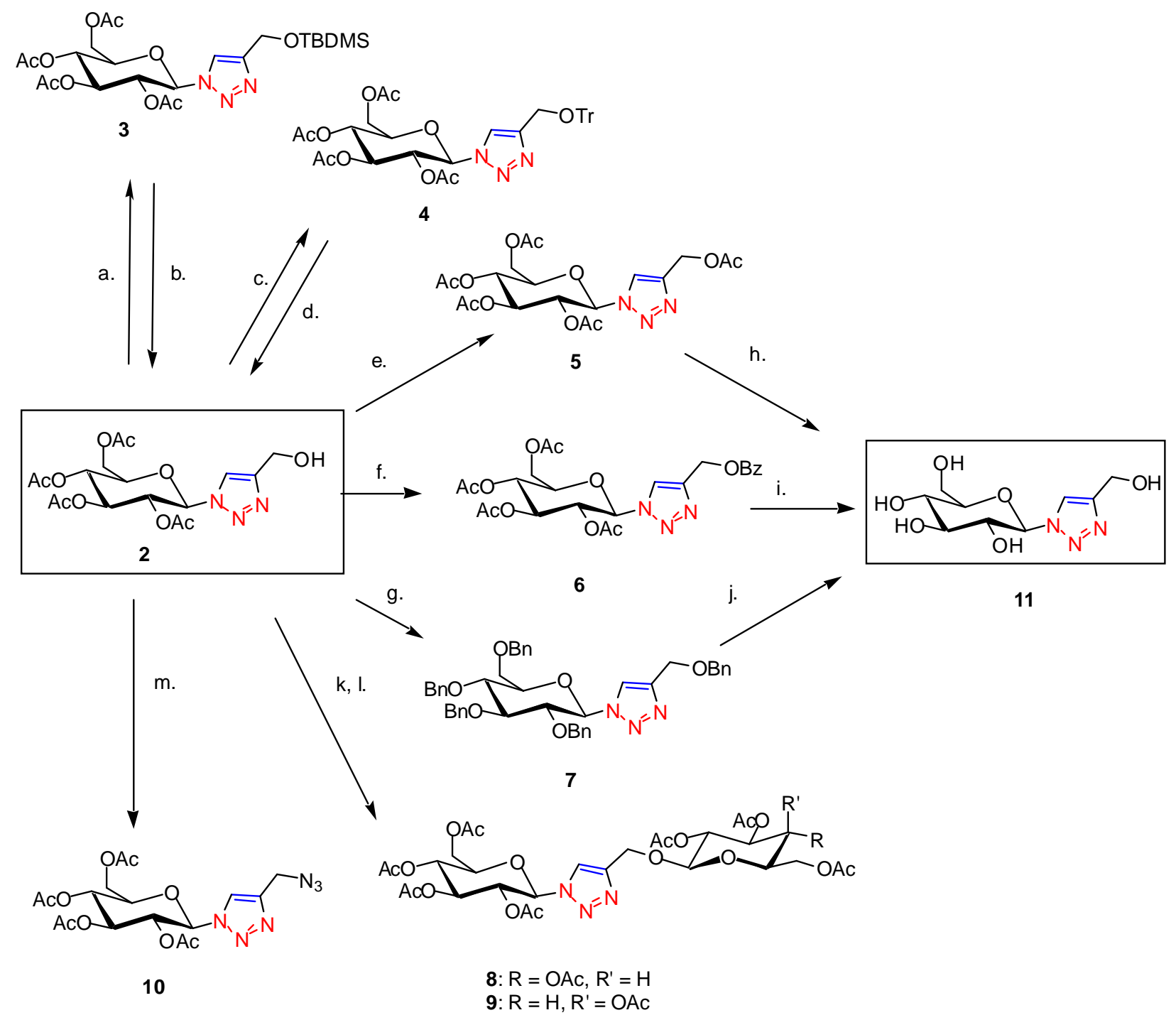

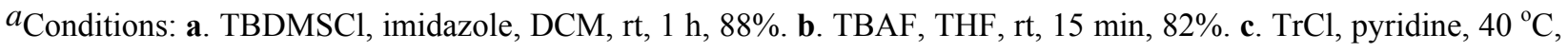
o/n, 90\%. d. camphor sulfonic acid, DCM, rt, 4 h, 85\%. e. $\mathrm{Ac}_{2} \mathrm{O} /$ pyridine, rt, 2 h, 90\%. f. $\mathrm{BzCl}_{1} \mathrm{Et}_{3} \mathrm{~N}, \mathrm{DCM}, \mathrm{o} / \mathrm{n}$, $82 \%$. g. i) $\mathrm{NaOCH}_{3}, \mathrm{CH}_{3} \mathrm{OH}$, rt, 2 h; then ii) $\mathrm{NaH}, \mathrm{BnBr}, \mathrm{DMF}$, rt, o/n, $68 \%$. h. $\mathrm{NaOCH}_{3}, \mathrm{CH}_{3} \mathrm{OH}$, rt, 30 min, $90 \%$. i. $\mathrm{NaOCH}_{3}, \mathrm{CH}_{3} \mathrm{OH}, \mathrm{rt}, 12$ h, 92\%. j. $\mathrm{H}_{2}, 30 \% \mathrm{Pd}(\mathrm{OH})_{2} / \mathrm{C}, \mathrm{CH}_{3} \mathrm{OH} / \mathrm{DCM}$, rt, 24 h, 78\%. k. 2,3,4,6-tetra-O-acetyl- $\alpha$-Dglucopyranosyl bromide (1.0 equiv), AgOTf, DCM, $52 \%$ of 8. $\mathbf{l}$. $\beta$-D-galactose pentaacetate (1.0 equiv), $\mathrm{BF}_{3}$. $\mathrm{Et}_{2} \mathrm{O}$, DCM, rt, o/n, 43\% of 9. m. i) MsCl, Et 3 N, DCM, rt, 30 min; then ii) $\mathrm{NaN}_{3}, \mathrm{DMF}, \mathrm{o} / \mathrm{n}, \mathrm{rt}, 53 \%$. 
Chemical structure of compound $\mathbf{2 0}$

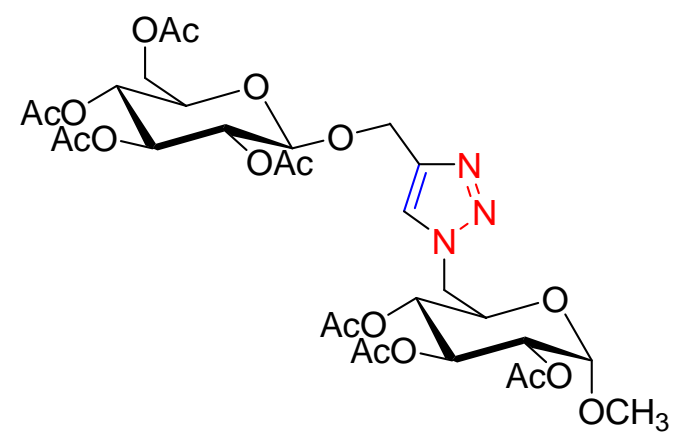

20 